\title{
Seller Reputation and Trust in Pre-Trade Communication*
}

\author{
BRUno Jullien \\ Toulouse School of Economics \\ Toulouse, France
}

\author{
IN-UCK PARK \\ University of Bristol \\ Bristol, U.K.
}

September 28, 2009

\begin{abstract}
It is shown that if there is adverse selection on seller's ability in experience goods market, credible communication can be sustained by reputation motives in spite of the inherent conflict of interests between sellers and buyers. In the absence of "commitment" types, reputation motives are explained as a consequence of equilibrium interplay between the market's perception on a seller's ability to deliver quality and the level of trust it places on the information he provides. Moreover, reputation motives do not disappear even after the seller's ability is revealed. This model is applied to examine the extent to which consumer rating systems may discipline sellers in honestly informing buyers about the quality of their product. Also analyzed is the impact of the possibility that sellers may restart as new traders by obtaining new identities. (JEL Codes: C73, D82, D83, L14)
\end{abstract}

Keywords: cheap talk, consumer rating system, reputation, trust.

\section{Introduction}

It is widely recognized that reputational concerns help to sustain the trust that is essential in effective transactions of experience goods. The problem of potential inefficiencies due to hidden information and moral hazard is particularly acute in service industries and internet markets. ${ }^{1}$ As an attempt to overcome this problem, many trading websites adopt consumer rating systems, but their effectiveness is far from proven. ${ }^{2}$ In light of the rapid expansion of these markets,

${ }^{*}$ The research is supported by the Leverhulme Trust, and was initiated while B. Jullien was visiting the University of Bristol. The authors thank Heski Bar-Isaac, Luis Cabral, Yuk-Fai Fong, David Levine, Thomas Mariotti, Jérome Mathis, Andrew McLennan, Fabien PostelVinay, Ron Siegel, Joel Sobel, Peter Sorensen, and Mike Whinston for useful discussion and comments, as well as seminar participants at Oxford University, the University of Naples, and the CSIO/IDEI Workshop in Toulouse School of Economics. Emails: bjullien@cict.fr and i.park@bristol.ac.uk.

${ }^{1}$ See Dellarocas (2003 and 2005) and references therein for a discussion of reputation issues on Internet, and Bar-Isaac and Tadelis (2006) for a survey of the literature on seller reputation.

${ }^{2}$ Various studies find marginal effect, e.g., Jin and Kato (2006). See also a survey paper by Bajari and Hortacsu (2004) and the references therein. A recent study of eBay data by 
this paper is an attempt to further our understanding of the key factors that determine the effectiveness of reputation mechanisms in market environments.

For a seller of goods or services, reputation can encompass several dimensions. In particular, it reflects the beliefs on his "ability" to deliver a good/service of high quality. In addition, it may also reflect the level of "trust" attached to the soft information provided by the seller prior to transaction, concerning the quality, suitability, etc., of the particular item for trade. These two dimensions of reputation are undoubtedly interrelated.

We present a model that delineates the mechanism and effect of this interrelation. Since trustworthy behavior implies a short-term sacrifice, it is worth pursuing only if it is expected to bring enough future reward. In an efficient market in which sellers are paid according to the expected quality of the items they deliver, such a future reward can take the form of an enhanced perception of the market on the seller's ability to deliver high quality, which is typically referred to as the seller's reputation. Of course trustworthy behavior will lead to an enhanced reputation only if sellers of high ability behave more trustworthily than those of low ability, lending an intrinsic link between the two dimensions of reputation, ability and trust, in market environments.

We show how such a link between ability and trust can emerge endogenously, steming from the fact that the cost of continuing with trustworthy behavior is larger for a low-ability seller who anticipates more "bad news" that she will have to disclose honestly. The incentives of a low-ability seller to mimic the trustworthy behavior of the high-ability sellers for future reputational rent eventually dissipates, leading to separation of sellers of different abilities which takes place when a seller's reputation grows above a certain level. Even after separation, high-ability sellers continue to preserve their reputation by resisting temptation to cheat.

In this equilibrium, credible communication (of a considerable degree, if not full) is sustained in spite of the inherent conflict of interests between sellers and buyers, owing to the combined effect of separation motive of high-ability sellers and mimicking motive of low-ability sellers. Thus, high-ability sellers are more reliable in disclosing nonverifiable information to buyers honestly. We note that adverse selection on seller's ability is indispensable for our result: If the seller's ability is publicly known, repeated interactions would not suffice for any credible communication to take place, however patient the seller may be. It may also be worth noting that adverse selection on an arbitrarily small difference in ability is enough to actuate credible communication via reputation motives if the seller is sufficiently patient.

Cabral and Hortacsu (2008) reports a clear effect on sales of the first negative feedback which appears to cause more frequent negative feedbacks with smaller effects; and it also reports an increase of negative feedbacks just before exit by low reputation sellers. These findings are qualitatively consistent with our analysis. 
At a theoretical level, this paper builds on the reputation literature initiated by Kreps and Wilson (1982) and Milgrom and Roberts (1982), ${ }^{3}$ with some nontrivial differences. In this literature reputation arises either when an agent strategically mimics a certain behavior so as to be pooled with agents of another type who are committed to that behavior (pooling reputation), or when an agent strategically diverts from a certain behavior so as not to be mistaken for another type who is committed to that behavior (separating reputation) as in Mailath and Samuelson (2001). As such, reputational incentives tend to be temporary in these models because their effect vanishes once the agent's type is revealed (e.g., by cashing in the reputation),${ }^{4}$ unless the agent's type is subject to continual random changes for reasons like ownership changes.

In our model, reputation arises from the presence of adverse selection on seller's ability (in supplying good quality items), without the existence of a "commitment" type. In particular, both types of sellers are strategic and have conflicting interests with the buyer; however, the more reliable type (in delivering quality) always behaves trustworthily in equilibrium, while the less reliable type finds doing so worthwhile when his reputation is low but too costly when it is above a certain threshold.

Consequently, the motives for both the pooling and separating reputation coexist in our model, the former (latter) motive for the less (more) reliable type of seller. Moreover, reputational motives exist permanently in our model because agents of the more reliable type maintain trustworthy behavior even after they get separated from the less reliable counterparts and thus, their types revealed. ${ }^{5}$ It may be worth stressing that such behavior by the more reliable type is driven precisely by reputational concerns, because it is not viable without presence of the less reliable type as shown in Section 3.2.

Specifically, we model a situation where a seller randomly draws an item of either good or bad quality in each period and announces this quality as cheap talk. Each seller is of one of two private types, high or low ability: a high type

\footnotetext{
${ }^{3}$ Mailath and Samuelson (2006) provide an extensive review of the literature.

${ }^{4}$ Even under imperfect monitoring, Cripps et al. (2004) show that reputational motives disappear in the long-run because agents' types get revealed eventually.

${ }^{5}$ In most studies, the stage game payoff is independent of the reputation level in every period. Hence, the reputational behavior of normal/opportunistic type tend to be stationary under infinite-horizon perfect monitoring settings, exhibiting little dynamics of reputation building and cashing in. One way of generating such dynamics is by introducing the stochastic importance level of each period (Sobel, 1985). Another way is to relate the payoff of opportunistic behavior to the reputation level in a natural manner, such as in our paper and Benabou and Laroque (1992). In these cases, a less reliable agent is bound to cheat given a chance if he had been lucky enough in the past to have built up his reputation above a certain threshold, thus revealing the type. The logic of Cripps et al. (2004) is somewhat different: A long enough history of an agent's past behavior reveals his type with a statistically overwhelming confidence and consequently, he would indulge in opportunistic actions because each such action makes only a negligible dent on his reputation.
} 
seller draws a good quality item more frequently. Each item is traded at a price that is equal to the expected quality based on the seller's prevailing reputation and his announcement. The buyer learns the true quality and publicly reveals the truthfulness of the seller's announcement (e.g., ratings in online markets), which updates the seller's reputation level accordingly.

Without communication, the model gives rise to learning over time through observation of past quality, and prices reflect the evolution of beliefs on the seller's ability but not the true quality of the item for sale. Communication allows equilibria in which some information on the item's quality is credibly transmitted by the seller.

Our analysis focuses on equilbria in which high type sellers always announce truthfully, and we establish that there is a unique equilibrium of this kind. In this equilibrium each and every truthful announcement increases the seller's reputation, which has the effect of increasing the price he receives in the next period if he claims his item to be of a good quality. Low type sellers of all reputation levels falsely claim bad quality items to be good with a positive probability for short-term gain, after which their reputation vanishes. The probability of lying by a low type seller is a continuous but non-monotonic function of the prevailing reputation level.

Compared with the case without communication, this equilibrium exhibits faster learning of the seller's type and more information is incorporated in the price of the item. It thus mitigates the lemon's problem substantially.

As is often speculated, the reputation mechanism in online markets may be undermined due to the possibility that a trader may restart by obtaining a new identity after damaged reputation (in the same or another marketplace). We also analyze a model in which such restarts are possible and characterize stationary equilibrium. In particular, we show that this option increases cheating incentives by limiting the damage from abusing reputation and as a result, the probability that a low type seller lies is higher than when fresh restarts are infeasible, uniformly across all reputation levels.

Our paper contributes to the literature on reputation pioneered by Kreps and Wilson (1982) and Milgrom and Roberts (1982), and further developed by Diamond (1989), Fudenberg and Levine (1989), Mailath and Samuelson (2001), Ely and Valimaki (2003), and Cripps, Mailath and Samuelson (2004), among others. More specifically, it contributes to the literature on cheap-talk reputation which we briefly describe below. Due to the nature of the issue, it often concerns experts/advisors and certifiers. Sobel (1985) shows that an "enemy" (an informed agent who has a completely opposing preference to the decision maker) may build a reputation by mimicking the honest reporting of a "friend" (who has a perfectly aligned preference with the decision maker). Generalizing this model to noisy information, Benabou and Laroque (1992) study the reputa- 
tion of financial experts. In a model where an enemy is biased in one direction, Morris (2001) shows that even a friend may have a reputational incentive to lie. Ottaviani and Sorensen $(2001,2006)$ study reputational cheap talk in a different model where experts are motivated by exogenous payoff that increases in their perceived ability, akin to the career concerns literature. ${ }^{6}$ A recent paper by Mathis, McAndrews and Rochet (2009) examines the extent to which reputation concerns discipline rating agencies. ${ }^{7}$

As mentioned earlier, our reputation mechanism does not rely on the existence of an inherently honest type, unlike many papers mentioned above. Also, in contrast to Sobel (1985) and Morris (2001), a "friendly" seller cannot exist in our model because sellers have the same monotonic preferences over prices, i.e., both types of sellers are "enemies." As a consequence, knowing the seller's type would exacerbate the communication problem for all types in our context, whilst it would solve the problem for the friendly type in theirs. In this respect, our contribution differs substantially from theirs.

Bar-Isaac (2003) studies a model similar to ours but without the scope of pre-trade communication because even the seller does not observe the quality. In a context where the seller incurs a cost to trade in each period, he shows that the seller's decision to continue to trade is a signal that facilitates learning process and thus, enhances the seller's reputation. In contrast to our model, though, lacking means to cash in their reputation by duping buyers, both types of sellers benefit in the same way from high reputation and thus, do not separate when reputation is above a certain threshold.

Mailath and Samuelson (2001) analyze a moral hazard version of our model without pre-trade communication and show, inter alia, that for reputation effects to arise the seller's type needs to be subject to continual random changes. Our results extend to the case of moral hazard (see Section 6) and thus, suggest that pre-trade communication may restore reputation effects in their model even if the seller's type is fixed through time.

Finally, the current paper also makes a methodological innovation in establishing existence and uniqueness of the equilibrium when Blackwell's condition for a contraction mapping does not apply, as detailed in Section 4.2.

The next section describes the main model and defines equilibrium. Section 3 presents some preliminary results. Section 4 analyzes the reputation mechanism and characterizes the unique reputation equilibrium in which the high type sellers always trade truthfully. Section 5 analyzes an extended model in which

\footnotetext{
${ }^{6}$ Although the mechanism differs for experts, they also find that often adverse selection on experts' quality enhances meaningful communication.

${ }^{7}$ These papers use the adverse selection approach to reputation. Papers (on cheap-talk reputation) also exist that use the so-called "bootstrap" approach based on the folk theorem argument, e.g., Park (2005) on cheap talk reputation of differentiated experts and McLennan and Park (2007) on auditor reputation.
} 
sellers may opt out or restart with a new identity. Section 6 discusses some further extensions. Appendix contains some technical details.

\section{Model}

We consider a single marketplace (or website) where sellers of different abilities interact with a large set of buyers. There are infinite periods $t=1,2, \cdots$, and a representative seller is either of a high type $(\theta=h)$ or a low type $(\theta=\ell)$ where $0<\ell<h<1$. The seller's type $\theta \in\{h, \ell\}$ is private information. The seller's perceived ability in each period $t$ is captured by his reputation $\mu_{t} \in[0,1]$, the common belief that the prospective buyers attach to the seller being of a high type at the beginning of that period.

In each period $t$, a seller with reputation $\mu_{t}$ draws one item for sale of a random quality $q_{t}$ which is good $(g)$ with probability $\theta$ and bad $(b)$ with probability $1-\theta$ where $\theta \in\{h, \ell\}$ is the seller's type. We normalize as $g=1$ and $b=0$. Observing the quality of the item, the seller publicly makes a cheap talk announcement $m_{t} \in\{G, B\}$ about its quality, where $m_{t}=G(B)$ is interpreted as announcing the quality to be $g(b) .{ }^{8}$ We say that the agent lies if he announces $B$ when $q_{t}=g$ or $G$ when $q_{t}=b$, and tells the truth if he announces $G$ when $q_{t}=g$ or $B$ when $q_{t}=b .^{9}$

The prospective buyers are myopic and try to maximize the expected quality minus the price paid. We assume a competitive demand side so that each item is traded at a price that is equal to the expected quality ${ }^{10}$ calculated, a la Bayes rule, based on $\mu_{t}$ and the seller's equilibrium strategy of announcing $m_{t}$. At the end of the trading period, the purchaser observes the true quality $q_{t}$ and honestly reports it publicly. ${ }^{11}$ The seller's reputation is revised from $\mu_{t}$ to $\mu_{t+1}$ based on $m_{t}$ and $q_{t}$, and the period $t+1$ starts. The seller's objective is to maximize the discounted sum of its revenue stream with discount factor $\delta \in(0,1)$. At any date $t$, the full history of messages and items' quality of the seller is publicly known. The structure of this game, denoted by $\Gamma$, is common knowledge.

Our equilibrium concept is Markov perfect equilibrium, i.e., we focus on Perfect Bayesian equilibria such that the equilibrium strategies in each period

\footnotetext{
${ }^{8}$ Alternatively, we may model that each seller posts a price $p$ at which buyers either buy or not, and the purchaser of the item reports whether satisfied $(q \geq p)$ or not $(q<p)$. Our equilibrium continues to be an equilibrium in this alternative model. We don't consider the possibility that the seller announces his type $\theta$, although we conjecture that this would not change our results.

${ }^{9}$ Of course the labelling of the messages is somewhat arbitrary, but it will be unambiguous when we introduce the reputation equilibrium.

${ }^{10}$ This is in line with Mailath and Samuelson (2001) and Bar-Isaac (2003).

${ }^{11}$ We assumed that the quality, although observable by the buyer, cannot be verified ex-post, so that no warranty contract is feasible.
} 
depends only on the seller's reputation level of that period. Thus, the seller's equilibrium strategy is represented by two functions $x^{*}(\mu, q)$ and $y^{*}(\mu, q)$ that denote, respectively, the probability that a seller of $h$-type and $\ell$-type lies contingent on the prevailing reputation level $\mu \in[0,1]$ and the quality $q \in\{g, b\}$ of the item drawn.

Given $x^{*}(\mu, q)$ and $y^{*}(\mu, q)$, a "price/quality profile" $p_{m}^{*}(\mu)$ is defined as the posterior probability that the item is of a good quality $(q=g)$ when the seller with a reputation level $\mu$ announced $m \in\{G, B\}$, obtained by Bayes rule from the seller's strategy whenever possible. Being the expected quality, $p_{m}^{*}(\mu)$ is also the price at which the item will be traded.

A "transition rule" is a function $\pi_{m q}^{*}(\mu)$ that specifies the posterior probability that $\theta=h$ in the next period, when in the current period $\operatorname{Pr}(\theta=h)=\mu$ and the seller sells an item of quality $q \in\{g, b\}$ after announcing $m \in\{G, B\}$. We require that $\pi_{m q}^{*}(\mu)$ be obtained by Bayes rule from the seller's strategy whenever possible.

Given $x^{*}(\mu, q), y^{*}(\mu, q), p_{m}^{*}(\mu)$, and $\pi_{m q}^{*}(\mu)$ as above, we define the value function for $\theta \in\{h, \ell\}$, denoted by $V_{\theta}^{*}(\mu):[0,1] \rightarrow \mathbb{R}$, as the expected discounted sum of revenue stream of a seller of type $\theta$ and reputation $\mu$.

Definition 1 A collection $\left(x^{*}, y^{*}, p_{m}^{*}, \pi_{m q}^{*}, V_{\theta}^{*}\right)$ is a (Markov perfect) equilibrium if the followings hold for each $\theta=h, \ell$ where $z_{h}=x^{*}$ and $z_{\ell}=y^{*}$ :

(i) $z_{\theta}(\mu, g) \in \arg \max _{0 \leq z \leq 1}\left[z\left(p_{B}^{*}(\mu)+\delta V_{\theta}^{*}\left(\pi_{B g}^{*}(\mu)\right)\right)+(1-z)\left(p_{G}^{*}(\mu)+\delta V_{\theta}^{*}\left(\pi_{G g}^{*}(\mu)\right)\right)\right]$;

(ii) $z_{\theta}(\mu, b) \in \arg \max _{0 \leq z \leq 1}\left[z\left(p_{G}^{*}(\mu)+\delta V_{\theta}^{*}\left(\pi_{G b}^{*}(\mu)\right)\right)+(1-z)\left(p_{B}^{*}(\mu)+\delta V_{\theta}^{*}\left(\pi_{B b}^{*}(\mu)\right)\right)\right]$;

(iii) $V_{\theta}^{*}(\mu)=\theta\left[\left(1-z_{\theta}(\mu, g)\right)\left(p_{G}^{*}(\mu)+\delta V_{\theta}^{*}\left(\pi_{G g}^{*}(\mu)\right)\right)+z_{\theta}(\mu, b)\left(p_{B}^{*}(\mu)+\delta V_{\theta}^{*}\left(\pi_{B g}^{*}(\mu)\right)\right)\right]$ $+(1-\theta)\left[z_{\theta}(\mu, b)\left(p_{G}^{*}(\mu)+\delta V_{\theta}^{*}\left(\pi_{G b}^{*}(\mu)\right)\right)+\left(1-z_{\theta}(\mu, b)\right)\left(p_{B}^{*}(\mu)+\delta V_{\theta}^{*}\left(\pi_{B b}^{*}(\mu)\right)\right)\right]$.

Before turning to the characterization of the equilibria with adverse selection and cheap talk, we discuss a few properties of our model.

\section{Preliminary Considerations}

The term "reputation" in the economic literature encompasses several notions, two of which are present in our model. First, reputation may refer to the beliefs concerning the average quality provided by the seller to the market. In our model this corresponds to the beliefs $\mu_{t}$ on the seller's type $\theta$. Second, the notion of reputation may refer to the level of confidence that consumers have on the truthfulness of the announcement of the seller concerning the quality of the good. This notion thus refers more to trust than to beliefs on the type. As shown below, however, the two concepts are closely related. 
We use the term "learning" to refer to the fact that the mere observation of the history of quality $q_{t}$ helps consumers improve their knowledge on the seller's type, in a non-strategic manner.

\subsection{The learning equilibrium}

Suppose that there is no communication, say because the seller doesn't observe the quality of the good. Then, in every period a seller's item is traded at a price equal to the expected quality

$$
p_{t}=E\left(q \mid \mu_{t}\right)=\mu_{t} h+\left(1-\mu_{t}\right) \ell .
$$

In this case buyers' belief on a seller's type evolves according to the simple Bayes rule:

$$
\begin{aligned}
\mu_{t+1} & =\frac{\mu_{t} h}{\mu_{t} h+\left(1-\mu_{t}\right) \ell}>\mu_{t} \text { if } q_{t}=g \\
\mu_{t+1} & =\frac{\mu_{t}(1-h)}{\mu_{t}(1-h)+\left(1-\mu_{t}\right)(1-\ell)}<\mu_{t} \text { if } q_{t}=b .
\end{aligned}
$$

Beliefs and prices follow a martingale, so that the price increases or declines depending on whether the quality delivered last period was good or bad.

Notice that this equilibrium remains as an equilibrium in the game $\Gamma$ described in Section 2, i.e., the so-called "babbling equilibrium." For instance such an equilibrium obtains when the seller always announces $G$ and thus, the message $m_{t}$, containing no information content, is ignored. The beliefs and the price evolve as in the learning equilibrium above and since announcement doesn't affect the continuation game, it is trivially optimal for the seller to announce $G$.

\subsection{A single type}

Now suppose that there is a single type, say type $\ell$. (As we shall see below, this is different from saying that the buyers' beliefs assign probability 1 to $\theta=\ell$.) Due to risk-neutrality and certainty to trade, our model has the feature that there is a zero value for the seller of transmitting information to the buyer in this case. The reason is that the ex-ante payoff is equal to the expected price which always coincides with the expected quality. An implication is that repeated interaction cannot help to foster communication in this set-up.

To see this, consider any equilibrium of our game when a seller's type is publicly observed to be $\ell$. Because the price is the expected value conditional on the information available at date $t$, the ex-ante expected price must be equal to $\ell$. Thus, any equilibrium generates an expected payoff of $\frac{\ell}{1-\delta}$.

Another consequence is that there cannot be any information transmitted through communication. To see this, consider any period and suppose that the 
message is informative in that period. This would mean that the probability $y^{*}(b)$ of announcing $m=G$ when $q=b$ is not equal to the probability $1-y^{*}(g)$ of announcing $G$ when $q=g$. Then, the prices would differ for the two messages. But we have seen that the expected payoff from the next period on must be equal to $\frac{\ell}{1-\delta}$ independently of the message to be sent. Hence, the seller would announce with certainty the message that would generate the highest price, irrespective of $q$, which would contradict $y^{*}(b) \neq 1-y^{*}(g)$.

Thus, when the type of the seller is publicly observed, the unique equilibrium outcome is the no communication equilibrium outcome.

\subsection{On communication in equilibrium}

We say that an equilibrium involves communication if there is a positive probability that at some date the message conveys some information. In our model, there are two types of information that can be transmitted: information about the current level of quality, $g$ or $b$, and information about the type $\theta$.

Before we turn to the equilibrium analysis it is worth noting that the two types of information transmission are related in a non-trivial way. In our set-up, some information about $\theta$ will be transmitted by messages if

$$
x^{*}\left(\mu_{t}, q_{t}\right) \neq y^{*}\left(\mu_{t}, q_{t}\right)
$$

with a positive probability in some period $t$. To see this, observe that if the strategy of the seller is independent of his type then it must be the case that the posterior $\mu_{t+1}$ depends only on the history of the realized quality $\mathbf{h}^{t}=\left(q_{1}, \cdots, q_{t}\right)$ and not on the history of message $\mathbf{m}^{t}=\left(m_{1}, \ldots, m_{t}\right)$, i.e., $\operatorname{Pr}\left(\theta \mid \mathbf{h}^{t}, \mathbf{m}^{t}\right)=\operatorname{Pr}\left(\theta \mid \mathbf{h}^{t}\right)$. Similarly, some information about the product quality $(q)$ will be transmitted if $p_{G}^{*}\left(\mu_{t}\right) \neq p_{B}^{*}\left(\mu_{t}\right)$ with a positive probability and both messages may be sent with positive probability in some period.

We asserted above that communication about the quality of the item is not possible if there is a single type. This observation extends to the following property when there are multiple types, i.e., in a setting of adverse selection:

Property: Messages cannot convey information on the quality of the good unless they convey information on the type of the seller.

To see this, suppose that no information on $\theta$ is transmitted by messages in equilibrium. Then, updated beliefs would be a function only of the history of quality as argued above, which would imply that in any period the expected future payoff of a seller is independent of the current message to send. If $p_{G}^{*}\left(\mu_{t}\right) \neq p_{B}^{*}\left(\mu_{t}\right)$, therefore, both types of seller would send the same message with probability 1 (the one that fetches a higher price), which would dictate that the messages be uninformative, i.e., $p_{G}^{*}\left(\mu_{t}\right)=p_{B}^{*}\left(\mu_{t}\right)$, a contradiction. 
Therefore, adverse selection and signalling about the type are necessary ingredients for messages to be a credible signal of quality in our environment.

\section{Reputation Equilibrium}

We now turn to analysis of equilibrium with reputation. As we wish to study the extent to which reputation motives help to induce truthful revelation of the quality of the product, we focus on equilibria with the following properties:

(a) an $h$-type seller always tells the truth regardless of $q$ so long as $\mu>0$, i.e., $x^{*}(\mu, q)=0$ for all $\mu>0$; and

(b) the value function $V_{\theta}^{*}(\mu)$ is non-decreasing in $\mu$ for $\theta=h, \ell$.

The first property lends the idea that beliefs about the types will generate trust in messages. The intuition behind this property is that building/maintaining reputation through truthful announcement of the quality is less costly for an $h$-type seller because he knows he will have more good draws than an $\ell$-type seller, whence he should announces the truth with a larger probability.

The property (b) states that a seller's expected profit increases with the market's belief about his type. Notice that the expected quality of the product, $\mu h+(1-\mu) \ell$, increases with $\mu$. This would be the payoff of a seller in a one-shot game or if $\delta=0$. The property states that this monotonicity property extends to our dynamic setting, which seems natural.

We refer to such an equilibrium as a non-trivial reputation (NTR) equilibrium, to distinguish from the evolution of beliefs due to pure learning:

Definition 2 An equilibrium satisfying (a) and (b) is called a NTR-equilibrium.

In characterizing the equilibrium, we proceed in three steps. First, we derive some useful properties of the equilibrium. Then, we use these properties to prove existence, continuity and uniqueness of the value function for an $\ell$-type seller. Finally, we show that telling the truth is the optimal strategy for an $h$-type seller.

\subsection{Some properties of NTR-equilibria}

A first trivial remark is that it is not possible that an $\ell$-type seller always tells the truth. If he were to always tell the truth, the equilibrium price would be $p_{G}^{*}(\mu)=1$. Then, upon drawing a low quality item, by falsely reporting as good $(m=G)$ he would get a payoff of at least $1+\delta \ell /(1-\delta)$ because in any future period there is a message that would get a price at least $\ell$ (since the expected 
quality in the market is equal to some weighted average of $p_{G}^{*}$ and $p_{B}^{*}$ ), which is better than the expected payoff from telling the truth, $\delta \ell /(1-\delta)$. Thus, full revelation of $q$ by both types of seller is not possible.

Nonetheless, due to property (b) there should be no incentive to misreport good quality as bad, since this would reduce the current price without enhancing next period's reputation. We first show that this is indeed the case:

Lemma 1 In any NTR-equilibrium,

$$
y^{*}(\mu, g)=0 \quad \text { and } \quad p_{B}^{*}(\mu)=0 \quad \forall \mu \in(0,1] .
$$

Proof. First, we show that $p_{G}^{*}(\mu) \geq p_{B}^{*}(\mu)$ for all $\mu>0$. If $y^{*}(\mu, b)=0$, then $p_{G}^{*}(\mu)=1>p_{B}^{*}(\mu)$ is immediate by property (a) and Bayes rule. If $y^{*}(\mu, b)>0$, then $p_{G}^{*}(\mu)+\delta V_{\ell}^{*}\left(\pi_{G b}^{*}(\mu)\right) \geq p_{B}^{*}(\mu)+\delta V_{\ell}^{*}\left(\pi_{B b}^{*}(\mu)\right)$ and $\pi_{G b}^{*}(\mu)=0<\pi_{B b}^{*}(\mu)$ by (a) and Bayes rule. These two inequalities, together with property (b), imply that $p_{G}^{*}(\mu) \geq p_{B}^{*}(\mu)$ as desired.

Now, to prove (1) by contradiction, suppose $y^{*}(\mu, g)>0$ for some $\mu>$ 0 , which would imply $\pi_{B g}^{*}(\mu)=0<\mu<\pi_{G g}^{*}(\mu)$ by (a) and Bayes rule and thus, $p_{G}^{*}(\mu)+\delta V_{\ell}^{*}\left(\mu^{\prime}\right) \leq p_{B}^{*}(\mu)+\delta V_{\ell}^{*}(0)$ where $\mu^{\prime}=\pi_{G g}^{*}(\mu)$. Hence, from (b) and $p_{G}^{*}(\mu) \geq p_{B}^{*}(\mu)$ shown above, we further deduce that $p_{G}^{*}(\mu)=p_{B}^{*}(\mu)$ and $V_{\ell}^{*}\left(\mu^{\prime}\right)=V_{\ell}^{*}(0)$. Since $\mu<\mu^{\prime}$, we would also have $V_{\ell}^{*}(\mu)=V_{\ell}^{*}(0)$ by (b). In addition, note that $y^{*}(\mu, b)>0$ because $y^{*}(\mu, b)=0$ would mean that $p_{G}^{*}(\mu)=$ $1=p_{B}^{*}(\mu)$, an impossibility. Hence, $V_{\ell}^{*}(\mu)=p_{G}^{*}(\mu)+\delta V_{\ell}^{*}(0)$. Then, since $V_{\ell}^{*}\left(\mu^{\prime}\right) \geq p_{m}^{*}\left(\mu^{\prime}\right)+\delta V_{\ell}^{*}(0)$ holds for either $m \in\{G, B\}$, the equality $V_{\ell}^{*}\left(\mu^{\prime}\right)=$ $V_{\ell}^{*}(\mu)$ obtained above would imply that $p_{m}^{*}\left(\mu^{\prime}\right) \leq p_{G}^{*}(\mu)$ must hold for both $m=G, B$. But, this is impossible because some weighted average of $p_{G}^{*}\left(\mu^{\prime}\right)$ and $p_{B}^{*}\left(\mu^{\prime}\right)$ is the expected quality of an item drawn by a seller of reputation $\mu^{\prime}$, hence must be strictly greater than $p_{G}^{*}(\mu)=p_{B}^{*}(\mu)=\mu h+(1-\mu) \ell$. Therefore, we have to conclude that $y^{*}(\mu, g)=0$ for all $\mu>0$.

Finally, $y^{*}(\mu, g)=0$ and (a) imply that $p_{B}^{*}(\mu)=0$, since message $m=B$ is sent with positive probability (at least by $h$-type) but only when $q=b$.

Observe that in equilibrium, once a seller's reputation falls to $\mu=0$, he cannot increase his reputation above 0 , because Bayes rule dictates that $\pi_{m q}^{*}(0)=0$ for any $m$ that is sent with a positive probability for quality $q$ at $\mu=0$. Therefore, a seller with reputation 0 announces the message that gives the highest price regardless of $q$, which implies that the seller gets the same equilibrium price, $\ell$, regardless of $q$. This is the case when an $\ell$-type seller's announcement strategy is independent of $q$ when $\mu=0$. Since labeling of the messages is inconsequential due to the costless nature of cheap talk messages, we make the convention that an $\ell$-type seller announces $G$ regardless of $q$ when $\mu=0$, i.e.,

$$
y^{*}(0, g)=0, y^{*}(0, b)=1 \quad \text { and } \quad p_{G}^{*}(0)=\ell, p_{B}^{*}(0)=0 \text {. }
$$


Next, it is straightforward to verify that payoffs at extreme beliefs coincide with those under full information:

Lemma 2 In any NTR-equilibrium, $V_{\ell}^{*}(0)=\frac{\ell}{1-\delta}$ and $V_{h}^{*}(1)=\frac{h}{1-\delta}$.

Proof. Consider an $\ell$-type seller with $\mu=0$. By (2) and Bayes rule, his reputation remains constant at 0 and he gets a price $p_{G}^{*}(0)=\ell$ in every period. Hence, $V_{\ell}^{*}(0)=\ell /(1-\delta)$. Next, consider an $h$-type seller with $\mu=1$. By property (a) and Bayes rule, his reputation remains constant at 1 and he gets $p_{G}^{*}(1)=1$ with a probability $h$ and $p_{B}^{*}(1)=0$ with a probability $1-h$ in every period. Hence, $V_{h}^{*}(1)=h /(1-\delta)$.

Having pinned down $y^{*}(\mu, g)=0$ for all $\mu$ as in (1) and (2) above, we now focus on the equilibrium values of $y^{*}(\mu, b)$. For notational ease, we use $y^{*}(\mu)$ as shorthand for $y^{*}(\mu, b)$ in the sequel. For any given $y^{*}(\mu)$, we define the equilibrium prices and transition rules as explained below.

For every $\mu, y \in[0,1]$, we define

$$
p_{G}(\mu, y):=\frac{\mu h+(1-\mu) \ell}{\mu h+(1-\mu)(\ell+(1-\ell) y)},
$$

the expected quality of the product claimed as good $(m=G)$ by a seller with reputation $\mu$ if an $\ell$-type seller would falsely claim so with probability $y$. In equilibrium it is equal to the price so that

$$
p_{G}^{*}(\mu)=p_{G}\left(\mu, y^{*}(\mu)\right)>0,
$$

whereas the price is $p_{B}^{*}(\mu)=0$ when the seller announces $B$ as asserted above. The following observations are straightforward from (3):

Property (I) For $y>0$ and $\mu<1, p_{G}(\mu, y)$ strictly increases in $\mu$ and strictly decreases in $y$, with $p_{G}(\mu, 0)=1$ for all $\mu, p_{G}(1, y)=1$ for all $y$, and $p_{G}(0,1)=\ell$.

We now consider beliefs. First, note that $\pi_{B g}^{*}(\mu)$ is not defined by Bayes rule because a seller never claims $m=B$ when $q=g$. We set $\pi_{B g}^{*}(\mu)=0$ without loss of generality: Since $V_{\theta}^{*}(\mu)$ is minimal at $\mu=0$, this does not affect incentive compatibility conditions $(i)$ of Definition 1.

Next, consider the beliefs when the seller announces truthfully. For all $(\mu, y) \neq(0,1)$, we define the following values:

$$
\begin{aligned}
\pi_{G g}(\mu) & :=\frac{\mu h}{\mu h+(1-\mu) \ell} \\
\pi_{B b}(\mu, y) & :=\frac{\mu(1-h)}{\mu(1-h)+(1-\mu)(1-\ell)(1-y)} .
\end{aligned}
$$


Note that $\pi_{G g}\left(\mu_{t}\right)$ is the Bayes-updated reputation level $\left(\mu_{t+1}\right)$ when a seller of reputation $\mu_{t}$ announced $m_{t}=G$ and the purchaser reported $q_{t}=g$; and $\pi_{B b}\left(\mu_{t}, y^{*}\left(\mu_{t}\right)\right)=\pi_{B b}^{*}\left(\mu_{t}\right)$ is that when a seller of reputation $\mu_{t}$ announced $m_{t}=$ $B$ and the purchaser reported $q_{t}=b$. Thus, we have

Property (II) For $y<1$ and $\mu<1, \pi_{G g}(\mu)$ and $\pi_{B b}(\mu, y)$ strictly increases in $\mu$, and $\pi_{B b}(\mu, y)$ strictly increases in $y$, with $\pi_{B b}(\mu, 1)=1$ and $\pi_{B b}(1, y)=1$.

Lastly, if a seller lies when $q=b$, the updated reputation level should be $\pi_{G b}^{*}(\mu)=0$ by Bayes rule, except when $\mu=1$ or $y^{*}(\mu)=0$. However, for $\mu<1$, there is no loss of generality in assuming that $\pi_{G b}^{*}(\mu)=0$ when $y^{*}(\mu)=0$ since, as before, this does not affect the incentive compatibility condition at $\mu<1$. (In addition, we will show shortly that $y^{*}(\mu)=0$ never happens.)

Determining $\pi_{G b}^{*}(1)$ is a little more delicate because it determines the value of $V_{\ell}^{*}(1)$ and thereby, the optimality of $y^{*}(\mu)=1$ for $\mu<1$ via determining the deviation value when an $\ell$-type seller with reputation $\mu$ announced truthfully upon drawing $q=b$, which would induce belief $\pi_{B b}(\mu, 1)=1$.

The value of $\pi_{G b}^{*}(1)$ also plays a central role for a seller of "maximal reputation" $\mu=1$, who, upon drawing $q=b$, has a choice between maintaining its reputation with a low current price $\left(p_{B}^{*}(1)=0\right)$, and a high current price of $p_{G}^{*}(1)=1$ followed by a drop of future profits from $V_{\theta}^{*}(1)$ to $V_{\theta}^{*}\left(\pi_{G b}^{*}(1)\right)$ due to lost reputation. The next lemma characterizes what happens for an $\ell$-type seller at $\mu=1$ in equilibrium.

Lemma 3 In any NTR-equilibrium,

(i) $y^{*}(1)=1$ and $y^{*}(\mu)$ is continuous at $\mu=1$;

(ii) $\lim _{\mu \rightarrow 1} V_{\ell}^{*}(\mu)=\bar{V}_{\ell}:=\frac{1-\delta\left(1-\ell+\ell^{2}\right)}{(1-\delta)(1-\delta \ell)}$;

(iii) if $y^{*}(\mu)<1$ for some $\mu$, then $V_{\ell}^{*}(1)=\bar{V}_{\ell}$ and $V_{\ell}^{*}\left(\pi_{G b}^{*}(1)\right)=V_{\ell}^{*}(0)$.

(iv) if $y^{*}(\mu) \equiv 1$, then there exists an NTR-equilibrium with the same seller's strategy (i.e., $\left.y^{*}(\mu) \equiv 1\right)$ and $\pi_{G b}^{*}(1)=0$ and $V_{\ell}^{*}(1)=\bar{V}_{\ell}$.

Proof. See Appendix.

The lemma implies that without loss of generality we can set $\pi_{G b}^{*}(1)=0$. This does not affect the value function $V_{\ell}^{*}$ due to Lemma 3 (iii) if an $\ell$-seller announces truthfully with some probability when $q=b$. In the case that an $\ell$-seller lies whenever $q=b$, there is some flexibility in setting the beliefs $\pi_{G b}^{*}(1)$ that would affect the value $V_{\ell}^{*}(1)$ but not the value function $V_{\ell}^{*}(\mu)$ for $\mu<1$. However, this is irrelevant for the equilibrium path since an $\ell$-seller never reaches the maximum reputation level $\mu=1$ in equilibrium. The value $V_{\ell}^{*}(1)$ solves $V_{\ell}^{*}(1)=1+\delta\left(\ell V_{\ell}^{*}(1)+(1-\ell) V_{\ell}^{*}(0)\right)$, yielding $\bar{V}_{\ell}$ in the lemma. 
In the sequel, therefore, we restrict attention to NTR-equilibria such that $\pi_{G b}^{*}(1)=0$. For such an NTR-equilibrium, we always have

$$
V_{\ell}^{*}(1)=V_{\ell}^{*}(0)+\Delta
$$

where

$$
\Delta:=\frac{1-\delta\left(1-\ell+\ell^{2}\right)}{(1-\delta)(1-\delta \ell)}-\frac{\ell}{1-\delta}=\frac{1-\ell}{1-\delta \ell}<1
$$

\subsection{Value and policy functions for $\ell$-type}

For any NTR-equilibrium, in light of Lemma 3 and the conditions (i)-(iii) of Definition 1 , an $\ell$-seller's value function $V_{\ell}^{*}$ is characterized by

$$
\begin{aligned}
V_{\ell}^{*}(\mu)= & \left(\ell+(1-\ell) y^{*}(\mu)\right) p_{G}\left(\mu, y^{*}(\mu)\right) \\
& +\delta\left[\ell V_{\ell}^{*}\left(\pi_{G g}(\mu)\right)+(1-\ell)\left(y^{*}(\mu) V_{\ell}^{*}(0)+\left(1-y^{*}(\mu)\right) V_{\ell}^{*}\left(\pi_{B b}\left(\mu, y^{*}(\mu)\right)\right)\right)\right]
\end{aligned}
$$

where

$$
y^{*}(\mu) \in \arg \max _{0 \leq y \leq 1} y\left(p_{G}\left(\mu, y^{*}(\mu)\right)+V_{\ell}^{*}(0)\right)+(1-y) \delta V_{\ell}^{*}\left(\pi_{B b}\left(\mu, y^{*}(\mu)\right)\right) .
$$

Observe from (7) that $V_{\ell}^{*}$ is a fixed point of a mapping determined by the RHS of (7) via a "best response" function $y^{*}$ that satisfies (8). We formally define this mapping with a view to applying a fixed point theorem on the set $\mathcal{F}$ of all non-decreasing functions $V:[0,1] \rightarrow \mathbb{R}$ such that $V(0)=\frac{\ell}{1-\delta}$ and $V(1)=\frac{\ell}{1-\delta}+\Delta$.

For a given $V \in \mathcal{F}$ we define the "pseudo-best-response" as a function $y_{V}$ : $[0,1] \rightarrow[0,1]$ that satisfies

$$
\left\{\begin{array}{lll}
y_{V}(\mu)=0 & \text { if } & p_{G}\left(\mu, y_{V}(\mu)\right)<\delta\left(V\left(\pi_{B b}\left(\mu, y_{V}(\mu)\right)\right)-V(0)\right) \\
y_{V}(\mu)=1 & \text { if } & \left.p_{G}\left(\mu, y_{V}(\mu)\right)>\delta\left(V\left(\pi_{B b}\left(\mu, y_{V}(\mu)\right)\right)\right)-V(0)\right) .
\end{array}\right.
$$

Since $p_{G}(\mu, y)-\delta V\left(\pi_{B b}(\mu, y)\right)$ is strictly decreasing in $y$, there exists a unique pseudo-best-response $y_{V}$ as verified below.

The value on the RHS of the inequalities in (9) is the gain from enhanced reputation that accrues to the seller when he truthfully announces a bad quality. Notice that it is bounded by $\delta \Delta<1$ by (6). This has two implications. First, since $p_{G}(\mu, 0)=1>\delta \Delta$, it must be the case that an $\ell$-seller lies with a positive probability if $q=b$, i.e. $y_{V}(\mu)>0$ for all $\mu$. Second,

$$
\bar{\mu}:=\inf \left\{\mu \in[0,1] \mid p_{G}(\mu, 1)>\delta \Delta\right\}<1
$$

where the inequality follows from $p_{G}(1,1)=1$. Then, it is immediate from Property (I) that $y_{V}(\mu)=1$ for all $\mu>\bar{\mu}$. Notice that the threshold $\bar{\mu}$ may be zero, in which case an $\ell$-type seller always lies whenever $q=b$. 
At the other end of the interval, because $\pi_{B b}(0, y)=0$ for all $y<1$, it must be the case that an $\ell$-seller lies with probability 1 if $q=b$ at $\mu=0$. Lastly, for $0<\mu<\bar{\mu}$, a best response $y \in(0,1)$ must satisfy

$$
\delta \lim _{y^{\prime} \uparrow y}\left(V\left(\pi_{B b}\left(\mu, y^{\prime}\right)\right)-V(0)\right) \leq p_{G}(\mu, y) \leq \delta \lim _{y^{\prime} \downarrow y}\left(V\left(\pi_{B b}\left(\mu, y^{\prime}\right)\right)-V(0)\right) .
$$

Consequently, the unique pseudo-best-response function $y_{V}$ is defined as

$$
y_{V}(\mu)= \begin{cases}1 & \text { if } \mu>\bar{\mu} \\ \text { the unique } y \text { that satisfies (10) } & \text { if } 0<\mu \leq \bar{\mu} \\ 1 & \text { if } \mu=0 .\end{cases}
$$

The properties of $y_{V}(\mu)$ are also characterized below.

Lemma 4 For any $V \in \mathcal{F}, y_{V}(\mu)$ is continuous and strictly positive on $[0,1]$ and $p_{G}\left(\mu, y_{V}(\mu)\right)$ is nondecreasing in $\mu$.

Proof. For each $\mu \in(0, \bar{\mu}]$, by construction, $y_{V}(\mu)$ is the value of $y$ at which the graph of $p_{G}(\mu, y)$ intersects with the "connected" graph of $\delta\left(V\left(\pi_{B b}(\mu, y)\right)-\right.$ $V(0)$ ), i.e., the latter graph is connected vertically at every discontinuity points by the shortest distance. Since both of the graphs are uniformly continuous as functions of $\mu$, the intersection point changes continuously in $\mu$, i.e., $y_{V}(\mu)$ is continuous on $\mu \in(0, \bar{\mu}]$. Since $p_{G}(\mu, 0)=1>\delta \Delta$ as argued above, the intersection takes place at some $y>0$, establishing that $y_{V}(\mu)>0$ for $\mu \in(0, \bar{\mu}]$ as well as when $\mu=0$ and $\mu>\bar{\mu}$ as per (11).

Furthermore, note that $y_{V}(\mu) \rightarrow 1$ as $\mu \rightarrow 0$ because, for every $y<1$, $\pi_{B b}(\mu, y) \rightarrow 0$ as $\mu \rightarrow 0$ and thus, $\delta\left(V\left(\pi_{B b}(\mu, y)\right)-V(0)\right)<\ell \leq p_{G}(\mu, y)$ for all $\mu$ sufficiently small. Since $y_{V}(\bar{\mu})=1$ by construction (using $y_{V}(0)=1$ if $\bar{\mu}=0$ ), it follows that $y_{V}(\mu)$ is continuous on $[0,1]$.

For $\mu \geq \bar{\mu}$, we have $p_{G}\left(\mu, y_{V}(\mu)\right)=p_{G}(\mu, 1)$ which increases in $\mu$ by Property (I). For $\mu \in(0, \bar{\mu})$, note that the two aforementioned graphs move upward as $\mu$ increases due to Property (I) and (II). Hence, the height of the intersection point also increases, i.e., $p_{G}\left(\mu, y_{V}(\mu)\right)$ weakly increases in $\mu$.

Finally, define a mapping $T: \mathcal{F} \rightarrow \mathcal{F}$ by

$$
T(V)(\mu):=p_{G}\left(\mu, y_{V}(\mu)\right)+\delta\left(\ell V\left(\pi_{G g}(\mu)\right)+(1-\ell) V(0)\right) .
$$

The next result establishes that the value function $V_{\ell}^{*}$ of an NTR-equilibrium can be computed as a fixed point of the operator $T$.

Lemma $5 T(\mathcal{F}) \subset \mathcal{F}$ and for any $N T R$-equilibrium, $V_{\ell}^{*}$ is a fixed point of $T$ and $y^{*}(\mu)=y_{V_{\ell}^{*}}(\mu)$. 
Proof. Since $\pi_{G g}(\mu)$ increases in $\mu$ and $p_{G}\left(\mu, y_{V}(\mu)\right)$ is non-decreasing in $\mu$ by Lemma 4 , it follows that $T(V)$, defined in (12), is non-decreasing in $\mu$.

Next, since $y_{V}(0)=1$ and $\pi_{G g}(0)=0$, we have $T(V)(0)=p_{G}(0,1)+$ $\delta(\ell V(0)+(1-\ell) V(0))$ which yields $T(V)(0)=\ell /(1-\delta)$.

Also, since $y_{V}(1)=\pi_{G g}(1)=1$ and $p_{G}(1,1)=1$, we have $T(V)(1)=1+$ $\delta(\ell V(1)+(1-\ell) V(0))$ which yields $T(V)(1)=\ell /(1-\delta)+\Delta$.

To prove that $V_{\ell}^{*}$ is a fixed point, first notice that the condition (8) implies that condition (9) is satisfied and thus that $y^{*}=y_{V_{\ell}^{*}}$. Since $y^{*}(\mu)=y_{V_{\ell}^{*}}(\mu)>0$ by Lemma 4, we deduce from (7) and (8) that $T\left(V_{\ell}^{*}\right)(\mu)=V_{\ell}^{*}(\mu)$ for all $\mu$.

Although $T: \mathcal{F} \rightarrow \mathcal{F}$ is well-defined, fixed point theorems may not be applied directly because $\mathcal{F}$ is not a compact set. This problem is resolved by showing that we can restrict attention to the set $\mathcal{F}^{r}$ of all right-continuous and non-decreasing functions on $[0,1]$, which is compact in the weak topology (see, e.g., Billingsley, 1999). We will then show that $T$ is continuous. For this we will need two key results.

The first result is continuity of the equilibrium value function.

Lemma 6 If $T(V)=V$ then $V$ is continuous and strictly increasing.

Proof. Since $y_{V}$ is continuous at 0 by Lemma 4, (12) implies that $V$ is continuous at 0 because

$$
\lim _{\mu \rightarrow 0} V(\mu)=\frac{p_{G}(0,1)+\delta(1-\ell) V(0)}{1-\delta \ell}=V(0) .
$$

Since $y_{V}(\mu)=1$ for all $\mu \geq \bar{\mu}$ and $\pi_{G g}(\mu)$ is continuous with $\pi_{G g}(1)=1$, we verify from (12) that $V$ is continuous at 1 as well:

$$
\lim _{\mu \rightarrow 1} V(\mu)=\frac{1-\delta\left(1-\ell+\ell^{2}\right)}{(1-\delta)(1-\delta \ell)}=V(1) .
$$

Next, since $y_{V}(\mu)$ is continuous in $\mu$ by Lemma 4 , (12) implies that

$$
\left|V^{+}(\mu)-V^{-}(\mu)\right| \leq \delta \max _{0<\mu<1}\left|V^{+}(\mu)-V^{-}(\mu)\right|
$$

holds for all $\mu \in(0,1)$ where $V^{+}(\mu)=\lim _{\mu^{\prime} \downarrow \mu} V\left(\mu^{\prime}\right)$ and $V^{-}(\mu)=\lim _{\mu^{\prime} \uparrow \mu} V\left(\mu^{\prime}\right)$. This implies that $\left|V^{+}(\mu)-V^{-}(\mu)\right| \equiv 0$ since $\delta<1$. Thus, $V$ is continuous.

Finally, it is clear from (12) that $V$ is strictly increasing on $(\bar{\mu}, 1)$ because $p_{G}(\mu, 1)$ is strictly increasing and $\pi_{G g}(\mu)$ is nondecreasing. If $V$ were not strictly increasing on $(0,1)$, one could find the highest value $\mu^{\prime} \leq \bar{\mu}$ such that $V(\mu)$ is constant on some interval $\left(\mu^{\prime}-\varepsilon, \mu^{\prime}\right)$. For sufficiently small $\varepsilon>0$, we would have $\pi_{G g}(\mu)>\mu^{\prime}$ if $\mu \in\left(\mu^{\prime}-\varepsilon, \mu^{\prime}\right)$ and thus, $V\left(\pi_{G g}(\mu)\right)<V\left(\pi_{G g}\left(\mu^{\prime}\right)\right)$. Since $p_{G}\left(\mu, y_{V}(\mu)\right)$ is non-decreasing by Lemma 4 , we would then have

$$
\begin{aligned}
V\left(\mu^{\prime}\right) & =p_{G}\left(\mu, y_{V}\left(\mu^{\prime}\right)\right)+\delta\left(\ell V\left(\pi_{G g}\left(\mu^{\prime}\right)\right)+(1-\ell) V(0)\right) \\
& >p_{G}\left(\mu, y_{V}(\mu)\right)+\delta\left(\ell V\left(\pi_{G g}(\mu)\right)+(1-\ell) V(0)\right)=V(\mu),
\end{aligned}
$$


contradicting the supposition that $V(\mu)$ is constant on $\left(\mu^{\prime}-\varepsilon, \mu^{\prime}\right)$. This proves that $V$ is strictly increasing.

The second result is a restriction on the evolution of beliefs in equilibrium.

Lemma 7 If $T(V)=V$, then $\pi_{B b}\left(\mu, y_{V}(\mu)\right)>\mu$ all $\mu>0$.

Proof. See Appendix.

Since it is easily verified that $\pi_{B b}\left(\mu, y_{V}(\mu)\right)>\mu$ if and only if $y>\hat{y}=\frac{h-\ell}{1-\ell}$, there is a uniform lower bound on the probability that $\ell$-type sellers misreport a bad quality.

Lemma 7 is key to our analysis because it implies that along any equilibrium path, the reputation level, and thus the price $p_{G}^{*}$, increases until the seller, in case he is of an $\ell$-type, reveals his type by falsely claiming a high quality, at which point the reputation level drops to 0 and the price to $\ell$. We are now ready to state our first main result.

Theorem 1 There exists a unique fixed point of $T$.

Proof. See Appendix.

As mentioned above, existence follows from the continuity of the operator $T$ on the set $\mathcal{F}^{r}$. The result thus differs from Benabou-Laroque (1992): In a model of financial experts who can manipulate the market by distorting information, they obtain existence and uniqueness by applying Blackwell's Theorem. ${ }^{12}$ This is not applicable to our model because our sellers may benefit by distorting information in only one direction, unlike their model where information holders can benefit by distorting the market in either direction, which generates symmetry in the model. More precisely, $T$ is not nondecreasing in $V$ and hence, Blackwell's condition for a contraction is not applicable. A second key difference is that Benabou and Laroque assume continuity whereas we do not restrict a priory to continuous functions.

Uniqueness is obtained separately from existence by using the properties of the fixed point. It stems from the observation that the value function is uniquely determined for $\mu>\bar{\mu}$. Using the fact that any truthful announcement enhances reputation along equilibrium path (Lemma 7), it is then possible to show that there is a unique way to "unravel" the value function by backward induction from the (stochastic) date where belief jumps above $\bar{\mu}$. A similar idea is exploited in Mathis, McAndrew and Rochet (2009) to obtain a constructive proof of existence in a model of rating agencies. Their proof relies on the fact that only positive claims generate trade and thus can be verified, which simplifies the analysis greatly. In our model both positive and negative claims can be verified.

\footnotetext{
${ }^{12}$ Morris (2001) and Bar-Isaac (2003) also use Blackwell's Theorem.
} 


\subsection{Optimality for $h$-type and equilibrium}

We have established that there is a unique pair of $V_{\ell}^{*}$ and $y^{*}(\mu)$ that is consistent with an NTR-equilibrium. The prices are then $p_{G}^{*}(\mu)=p_{G}\left(\mu, y^{*}(\mu)\right)$ and $p_{B}^{*}(\mu)=0$. We now establish the conditions under which truthful announcement is an equilibrium strategy for an $h$-seller and thus, an NTR-equilibrium exists. In doing so, without loss of generality we set the off-equilibrium beliefs as

$$
\pi_{B g}^{*}(\mu)=0 \leq \pi_{G g}(\mu) \quad \forall \mu \in[0,1]
$$

which ensures that reputation deteriorates after a false claim.

Let $V_{h}^{*}$ be the value function of an $h$-seller calculated from $x^{*}(\mu, q)=0$, given the strategy $y^{*}=y_{V_{\ell}^{*}}$ obtained for the unique fixed point $V_{\ell}^{*}$ of $T$, and prices $p_{m}^{*}$ and transition rules $\pi_{m q}^{*}$ defined for $y^{*}$. Recall that $x^{*}(0, q)$ has not been specified, yet. Thus, $V_{h}^{*}(0)$ is to be determined. Since $\pi_{G g}(0)=\pi_{B g}^{*}(0)=0$, however, $x^{*}(0, g)=0$ is clearly optimal.

For the moment we restrict attention to the cases that an $h$-seller lies with positive probability when $\mu=0$ and $q=b$, i.e., $x^{*}(0, b)>0$, so that

$$
V_{h}^{*}(0)=\frac{\ell}{1-\delta} \geq h \ell+\delta\left(h V_{h}^{*}(0)+(1-h) V_{h}^{*}\left(\pi_{B b}^{*}(0)\right)\right) .
$$

Later we will consider the possibility that $V_{h}^{*}(0)$ is larger, but this will not enlarge the set of parameters for existence.

Since $\pi_{B b}^{*}(0)$ is undefined by Bayes rule, we can ensure that (14) is satisfied by setting $\pi_{B b}^{*}(0)=0$, in which case $x^{*}(0, b)=1$ is an optimal strategy of an $h$-seller at $\mu=0$ when $q=b$. Higher values of $\pi_{B b}^{*}(0)$ subject to (14) would also work so long as optimality of $y^{*}(1)$ is retained, but they are inconsequential along the equilibrium path. Thus, we set $\pi_{B b}^{*}(0)=0$ without loss of generality.

Since $p_{G}(\mu, y)>\ell$ for all $\mu, y \in(0,1]$, an $h$-seller can guarantee a payoff exceeding $\ell /(1-\delta)$ in any continuation subgame by always claiming $q=g$. Thus, $V_{h}^{*}(\mu)>V_{h}^{*}(0)$ for all $\mu>0$, so it is clear from (13) that $x^{*}(\mu, g)=0$ is optimal for an $h$-seller for all $\mu>0$, as well as when $\mu=0$ as shown earlier.

Let us now consider $h$-seller's strategy when $q=b$. Once $\mu=1$ is reached, upon drawing $q=b$, an $h$-seller gets $\delta V_{h}^{*}(1)$ by reporting truthfully and $1+$ $\delta V_{h}^{*}(0)$ by reporting untruthfully because $\pi_{G b}^{*}(1)=0$ by Lemma 3 (iii) and Lemma 6 . Thus, it is optimal for an $h$-seller to report truthfully if and only if $\delta\left(V_{h}^{*}(1)-V_{h}^{*}(0)\right) \geq 1$ where $V_{h}^{*}(1)=h+\delta V_{h}^{*}(1)$, or equivalently,

$$
h-\ell \geq \frac{1-\delta}{\delta} \Longleftrightarrow \delta \geq \delta_{h}:=\frac{1}{h-\ell+1},
$$

which we assume below. It is clear that no NTR-equilibrium exists if (15) fails because the property (a) would not be satisfied. 
For $\mu>0$, we show $V_{h}^{*}(\mu)>V_{\ell}^{*}(\mu)$ holds below, based on the fact that an $h$-seller, if followed the same strategy as an $\ell$-seller, would obtain a higher expected payoff because he would get a better sequence of draws on average.

Lemma 8 If $V_{\ell}^{*}=T\left(V_{\ell}^{*}\right)$, then $V_{h}^{*}(\mu)>V_{\ell}^{*}(\mu)$ for all $\mu>0$.

Proof. See Appendix.

Upon drawing $q=b$ in any period with a prevailing reputation $\mu>0$, both types of seller would get the same expected payoff, $p_{G}\left(\mu, y^{*}(\mu)\right)+\delta V_{h}^{*}(0)$, from announcing $m=G$ by (14); but the payoff from announcing $m=B$ would be higher for an $h$-seller than for an $\ell$-seller according to Lemma 8 . Since an $\ell$-seller is indifferent between announcing $m=G$ and $m=B$ for $\mu \in(0, \bar{\mu})$, it follows that an $h$-seller must announce $m=B$ with probability 1 . For $\mu \geq \bar{\mu}$, an $h$ seller must announce $m=B$ for sure because the future gain from truth-telling, $\delta\left(V_{h}^{*}(1)-V_{h}^{*}(0)\right)$, exceeds the short-term gain of lying, $p_{G}\left(\mu, y^{*}(\mu)\right)$, by $(15)$. This leads to our second main result.

Theorem 2 There exists an NTR-equilibrium if and only if $\delta \geq \delta_{h}$. The equilibrium outcome is unique.

Proof. See Appendix.

To complete the characterization of NTR-equilibrium, we should point out that because the incentive constraint of $h$-seller is slack for $\mu>0$, there is the possibility that the value $V_{h}^{*}(0)$ differs from $V_{\ell}^{*}(0)$, which would be the case if $x^{*}(0, b)=0$. For this to hold in equilibrium, however, $\pi_{B b}^{*}(0)$ needs to be large enough for an $h$-seller to be willing to sacrifice a current profit $\ell$ to enhance his reputation to $\pi_{B b}^{*}(0)$, but not so large that an $\ell$-seller would want to do the same. A natural candidate would be the limit of $\pi_{B b}^{*}(\mu)$ as $\mu \rightarrow 0$. In addition, to extend the argument in the proof of Theorem 2 on incentive compatibility of $h$-type, we must preserve the property that the incentive to build reputation is higher for $h$-type than $\ell$-type, i.e., $V_{h}^{*}(\mu)-V_{h}^{*}(0) \geq V_{\ell}^{*}(\mu)-V_{\ell}^{*}(0)$. We show that this is the case if $h$ is large and the seller is patient enough.

Proposition 1 If $h>\frac{1+\sqrt{1+4 \ell^{2}+4 \ell^{3}}}{2+2 \ell}$ and $\delta$ is large enough, there exists an equilibrium such that $x^{*}(\mu, q)=0$ for all $\mu \in[0,1]$ and $q \in\{g, b\}$.

Proof. See Appendix.

\subsection{Discussion of the equilibrium}

In the unique equilibrium outcome, an $\ell$-seller lies with certainty when $q=b$ if $\mu \geq \bar{\mu}$. We may thus distinguish two types of equilibria depending on whether 
$\bar{\mu}$ is positive or zero. Notice that the threshold $\bar{\mu}$ is positive if $\delta$ is large enough so that

$$
\delta \Delta>\ell=p_{G}(0,1) \Longleftrightarrow \delta>\delta_{\ell}:=\frac{\ell}{1-\ell+\ell^{2}} .
$$

The following is immediate since $y_{V_{\ell}^{*}}(\mu) \in(0,1)$ was established for $\mu \in(0, \bar{\mu})$ from earlier discussion.

Corollary 1 If $\delta \leq \delta_{\ell}$, then $y^{*}(\mu)=1$ for all $\mu \in[0,1]$, while if $\delta>\delta_{\ell}$ then $y^{*}(\mu)<1$ for $0<\mu<\bar{\mu}$.

Thus, if $\delta_{h} \leq \delta \leq \delta_{\ell}$, all NTR-equilibria have a simple characterization: $\ell$ sellers always lie upon drawing a bad item (and are honest otherwise). ${ }^{13}$ Hence, the trading price $p_{t}$ increases over time until a bad draw occurs, at which point the type is revealed. Then, $p_{t}$ drops to $\ell$ for good if $\theta=\ell$; whilst $p_{t}=q_{t}$ in all subsequent periods if $\theta=h$, i.e., $p_{t}$ reflects the true quality.

If $\delta>\max \left\{\delta_{h}, \delta_{\ell}\right\}$, on the other hand, an $\ell$-seller with a reputation below $\bar{\mu}$ randomizes between announcing truthfully and untruthfully upon drawing $q=b$. As long as he tells the truth, he builds reputation and thus benefits from higher future prices for items he will announce to be good. Thus, reputation increases over time until one of two events occurs:

i) the seller falsely announces $m=G$ when $q=b$, in which case his type is revealed to be $\ell$ and the price drops to $\ell$ for good; or

ii) the seller truthfully announces $m=B$ when his reputation is $\mu>\bar{\mu}$, in which case his type is revealed to be $h$ and the price reflects the true quality in the future.

A salient characteristic of the NTR-equilibrium is that information on seller's type is revealed much faster than in the case without communication, where convergence occurs only asymptotically.

Corollary 2 In the NTR-equilibrium, the true type of seller is known in finite time with probability 1.

Proof. If a message is invalidated in some period, the type is known to be $\ell$. As long as messages are truthful, reputation increases so there is $\bar{t}$ such that $\mu_{t}>\bar{\mu}$ for sure if $t>\bar{t}$. Then if there were no lie before $\bar{t}$, the type is discovered in the first period $t>\bar{t}$ such that $q_{t}=b$, which occurs within a finite horizon with probability 1.

Thus, communication helps to mitigate the asymmetric information problem along two dimensions that are intrinsically related:

\footnotetext{
${ }^{13}$ In this case the equilibrium may vary in $V_{\ell}^{*}(1)$ and $\pi_{G b}(1,1)$ subject to $V_{\ell}^{*}(1)=1+$ $\delta\left(\ell V_{\ell}^{*}(1)+(1-\ell) V_{\ell}^{*}\left(\pi_{G b}(1,1)\right)\right)$, but they all generate the same equilibrium outcome.
} 
i) it helps credible communication of the true quality, thereby mitigating the lemon's problem;

ii) it helps consumers learn the true type of the seller.

Compared to the case of pure learning in Section 3.1, a key difference concerns updating of reputation following a bad draw $(q=b)$. In the learning equilibrium it is given by $\mu_{t+1}(b)=\pi_{B b}\left(\mu_{t}, 0\right)<\mu_{t}$. In the NTR-equilibrium reputation improves as long as the seller announces truthfully. Thus, while a bad quality is perceived as "bad news" when communication is not possible, it is perceived as "good news" in our model if truthfully announced.

The price dynamics also differ substantially. Along the NTR-equilibrium path, the price for items announced to be good increases over time roughly in line with the reputation until the seller's type is revealed, while the price stays constant at zero for items announced bad. In contrast, the price in the learning equilibrium follows a martingale where the price of date $t$ is independent of the realization of the quality at date $t$.

\section{Outside Option and New-life}

Up to now we have assumed that sellers stay in one marketplace forever and that memory is infinite. One of the issues surrounding the reputation mechanism based on consumer ratings is that sellers may find ways to escape from the bad consequences of damaged reputation. For instance, sellers may obtain an expected payoff above $V_{\ell}^{*}(0)$ by changing to another marketplace. Moreover, even within a given marketplace, it may be difficult to keep track of the identity of a seller, in which case a seller may have at any date an option to erase his history by changing his identity and start again as a new-comer. ${ }^{14}$ We examine the extent to which this possibility affects reputation mechanism.

\subsection{Equilibrium with an outside option}

First, consider the case that sellers have an option to leave the market at the end of any period, in which case he receives a fixed outside option, valued at $v_{o}$, in the subsequent period where $v_{o} \in\left(\frac{\ell}{1-\delta}, \frac{1}{1-\delta}\right)$. We characterize an NTR-equilibrium in this environment.

Recall that an NTR-equilibrium is one that satisfies properties (a) and (b). As before, ${ }^{15}$ it is characterized by the probability $y^{\dagger}(\mu)$ that an $\ell$-seller announces

\footnotetext{
${ }^{14}$ The ability to do so depends on the technology used by the platforms. This is known to be an issue with eBay for instance (see Delarocas 2006), but would be less of an issue when the platform controls the bank coordinates or the social status of companies, for then it would involve creating a new firm which is costly.

${ }^{15}$ The same argument as before shows that an $\ell$-seller truthfully announces when $q=g$.
} 
$m=G$ when $q=b$ and value functions $V_{\theta}^{\dagger}$ for $\theta \in\{h, \ell\}$. Then,

$$
y^{\dagger}(0)=1 \quad \text { and } \quad V_{\ell}^{\dagger}(0)=\ell+\delta v_{o} \in\left(\frac{\ell}{1-\delta}, v_{o}\right) .
$$

To see this, note that $V_{\ell}^{\dagger}(0) \geq p_{G}\left(0, y^{\dagger}(0)\right)+\delta v_{o}>\frac{\ell}{1-\delta}$. If $y^{\dagger}(0)<1$, then $V_{\ell}^{\dagger}(0)=\ell p_{G}\left(0, y^{\dagger}(0)\right)+\delta\left(\ell V_{\ell}^{\dagger}\left(\pi_{G g}(0)\right)+(1-\ell)\left(V^{\dagger}\left(\pi_{B b}\left(0, y^{\dagger}(0)\right)\right)\right) \leq \ell+\delta V_{\ell}^{\dagger}(0)\right.$, which would contradict $V_{\ell}^{\dagger}(0)>\frac{\ell}{1-\delta}$. Hence, we conclude that $y^{\dagger}(0)=1$. Thus, $V_{\ell}^{\dagger}(0)=p_{G}(0,1)+\delta \max \left\{v_{o}, V_{\ell}^{\dagger}(0)\right\}=\ell+\delta v_{o}$.

In addition, an argument analogous to the proof of Lemma 3 establishes, so long as $y^{\dagger}(\mu)<1$ for some $\mu$, that

$$
\lim _{\mu \rightarrow 1} y^{\dagger}(\mu)=y^{\dagger}(1)=1, \quad V_{\ell}^{\dagger}(1)=\frac{1+\delta(1-\ell) v_{o}}{(1-\delta \ell)}, \text { and } V_{\ell}^{\dagger}\left(\pi_{G b}^{\dagger}(1)\right) \leq v_{o} .
$$

Let

$$
\Delta_{v_{o}}:=\frac{1-(1-\delta) v_{o}}{(1-\delta \ell)}=V_{\ell}^{\dagger}(1)-v_{o} .
$$

Define $\mathcal{F}_{v_{o}}$ to be the set of all non-decreasing functions $V$ on $[0,1]$ such that $V(0)=V_{\ell}^{\dagger}(0)$ and $V(1)=V_{\ell}^{\dagger}(1)$. Define $y_{V}^{\dagger}(\mu)$ in the same manner as in (10) and (11) with $V(0)$ replaced by $v_{o}$ and $\bar{\mu}$ replaced by $\bar{\mu}^{\dagger}:=\inf \left\{\mu \mid p_{G}(\mu, 1)>\right.$ $\left.\delta \Delta_{v_{o}}\right\}<\bar{\mu}$ where the last inequality follows from $\delta \Delta_{v_{o}}<\Delta$. As long as $\delta \Delta_{v_{o}}>\ell$ so that $y_{V}^{\dagger}(\mu)<1$ for some $\mu$, which we assume below, we have $y_{V}^{\dagger}(\mu) \in(0,1)$ for $\mu \in\left(0, \bar{\mu}^{\dagger}\right)$ with $\lim _{\mu \rightarrow 0} y_{V}^{\dagger}(\mu)=1$ because $\delta\left(\max \left\{V\left(\pi_{B b}(\mu, y)\right), v_{o}\right\}-v_{o}\right)$ approaches $\delta \Delta_{v_{o}}>\ell$ as $y \rightarrow 1$ while it approaches 0 as $\mu \rightarrow 0$ for all $y<1$. Note that this implies

$$
V\left(\pi_{B b}\left(\mu, y_{V}^{\dagger}(\mu)\right)\right)-v_{o}>p_{G}\left(\mu, y_{V}^{\dagger}(\mu)\right) \quad \forall \mu \in\left(0, \bar{\mu}^{\dagger}\right) .
$$

Furthermore, $y_{V}^{\dagger}(\mu)$ is clearly continuous and assumes 1 for $\mu \geq \bar{\mu}^{\dagger}$. Define $T_{v_{o}}: \mathcal{F}_{v_{o}} \rightarrow \mathcal{F}_{v_{o}}$ as

$$
T_{v_{o}}(V)(\mu):=p_{G}\left(\mu, y_{V}^{\dagger}(\mu)\right)+\delta\left(\ell \max \left\{v_{o}, V\left(\pi_{G g}(\mu)\right)\right\}+(1-\ell) v_{o}\right) .
$$

It is straightforward to verify that $T_{v_{o}}(V) \in \mathcal{F}_{v_{o}}$.

Then, Lemmas 6 and 7 and Theorem 1 extend to $T_{v_{o}}$, establishing that, for any $v_{o} \in\left(\frac{\ell}{1-\delta}, \frac{1}{1-\delta}\right)$, there is a unique fixed point of $T_{v_{o}}$ and it is continuous and strictly increasing. We omit the proofs because they are analogous with straightforward changes due to the seller opting to restart whenever his reputation level is so low that the continuation value falls short of $v_{o}{ }^{16}$

\footnotetext{
${ }^{16}$ In the proof of lemma $7, V(\mu)=\sum_{t=0}^{\infty} \delta^{t} \ell^{t}\left(p_{G}\left(\pi_{G g}^{t}(\mu), y_{V}\left(\pi_{G g}^{t}(\mu)\right)\right)-\ell\right)+\frac{\delta v_{o}(1-\ell)+\ell}{1-\delta \ell}$, which implies that $V(\tilde{\mu})-v_{o}<\sum_{t=0}^{\infty}\left(p_{G}\left(\pi_{G g}^{t}(\tilde{\mu}), \hat{y}\right)-\ell\right) \delta^{t} \ell^{t}$ because $\frac{\delta v_{o}(1-\ell)+\ell}{1-\delta \ell}<v_{o}$.
} 
In the case that the outside option value is $v_{o}$ for an $h$-seller as well, optimality of truth-telling for $h$-type can be verified by an argument analogous to that leading to Theorem 2 , with $\delta_{h}$ replaced by the threshold $\delta_{v_{o}}$ that solves

$$
\delta_{v_{o}}\left(\frac{h}{1-\delta_{v_{o}}}-v_{o}\right)=1
$$

Thus, a unique equilibrium outcome exists if $\delta>\delta_{v_{o}}$ when sellers can exit for an outside option $v_{o}{ }^{17}$

If the outside option for the $h$-type differs from $v_{o}$, Theorem 1 is not valid. But the same reasoning as in the proof of Proposition 1 establishes existence of an NTR-equilibrium for sufficiently large $\delta$ so long as $h$-seller's outside option value is not too large.

To indicate their dependence on $v_{o} \in\left(\frac{\ell}{1-\delta}, \frac{1}{1-\delta}\right)$ with compact notations, let $y_{v_{o}}^{\dagger}$ and $V_{v_{o}}^{\dagger}$ denote the strategy of an $\ell$-type seller and the fixed point of $T_{v_{o}}$, respectively. Comparing with the findings in Section 4, we obtain

Proposition 2 For $v_{o} \in\left(\frac{\ell}{1-\delta}, \frac{1}{1-\delta}\right)$,

$$
y_{v_{o}}^{\dagger}(\mu)>y^{*}(\mu) \forall \mu \in(0, \bar{\mu}) \quad \text { and } \quad y_{v_{o}}^{\dagger}(\mu)=y^{*}(\mu)=1 \forall \mu \in[\bar{\mu}, 1] \text {. }
$$

Proof. In the Appendix.

Thus, availability of an option to exit and obtain an outside value larger than the value attached to bad reputation, results in a uniform increase in the probability that a bad item is falsely claimed as good. The consequence is a reduction in the price for items announced as good at all levels of reputation.

At the same time, note that learning takes place faster than in the equilibrium without such an option for two reasons. First, it is more likely that an $\ell$ seller reveals his type by falsely announcing $m=G$. Second, reputation gets updated to higher levels following truthful announcements. This also means that a reputation level is reached sooner at which the seller's type is revealed for sure if a bad item is drawn because an $\ell$-seller would definitely lie.

\section{$5.2 \quad$ New-life}

We now extend the analysis to the case that there is no outside option, but sellers can erase their history and start as new-comer at any date. To analyze

\footnotetext{
${ }^{17}$ Note that an $h$-seller with any reputation $\mu>0$ does not exit after trading a bad quality item because the value of updated reputation exceeds $v_{o}$ as (20) indicates. However, both types of seller may exit after trading a good quality item if the value of the updated reputation, $V_{\theta}^{\dagger}\left(\pi_{G g}(\mu)\right)$, falls short of $v_{o}$, although the seller would have exited already if this were to be the case.
} 
this environment, we need to put more structure on the dynamics behind the model because the incentive to change identity depends on the beliefs concerning new-comers, and these beliefs depend on equilibrium strategies.

We assume that there is a single platform on which trade can take place in any of infinite periods, where the past record of each seller is publicly known. There is a constant measure 1 of sellers on the platform in each period, although there is an exogenous entry/exit process. Each seller dies with probability $\chi \in(0,1)$ at the end of each period. These deaths are replaced by measure $\chi$ of new-born sellers at the beginning of the next period. Each new born seller is of $h$-type with probability $\mu_{i} \in(0,1)$.

If sellers cannot change identity, the model is the same as the one studied in Section 3 where the initial reputation starts at $\mu_{i}$ and the period $t$ is interpreted as the age or seniority of the seller (the number of trading periods since he joined the platform).

We suppose that a seller can start afresh with a new identity at any period. In this case he restarts the process as if he were a new-born seller, and we assume that it is not possible to distinguish a new-born seller from a old seller restarting afresh. We focus on stationary equilibria in which the proportion of $\ell$-sellers deciding to start afresh is constant, while $h$-sellers never lie and consequently, never change identity.

Thus all new sellers start with a reputation level set at an endogenous default level $\mu_{0} \in(0,1)$ which reflects the mix of genuine new-born sellers (of which a proportion $\mu_{i}$ are of type $h$ ) and equilibrium mass of sellers who restart. Let $v_{o}$ denote the value of an $\ell$-seller starting at the default reputation level $\mu_{0}$. Notice that the value function depends on $v_{o}$, while the mass $\mu_{0}$ depends on both the value function and $v_{o}$.

To endogenously determine $\mu_{0}$ and $v_{o}$, we start by treating $v_{o}$ as a parameter representing an outside option value to be obtained when a seller drops out of the market, so that we can apply the result from the previous section to determine the unique equilibrium strategy $y_{v_{o}}^{\dagger}$ and value function $V_{v_{o}}^{\dagger}$ of $\ell$-seller, that are consistent with $v_{o}$. Then, we calculate the value of $\mu_{0}$ accounting for the equilibrium mass of $\ell$-sellers who restart according to $y_{v_{o}}^{\dagger}$. Finally, we search for a fixed point $v_{o}$ that satisfies $v_{o}=V_{v_{o}}^{\dagger}\left(\mu_{0}\right)$.

To determine $\mu_{0}$ from $V_{v_{o}}^{\dagger}$ and $y_{v_{o}}^{\dagger}$, we calculate the mass of $\ell$-sellers who restart as explained below. Let $\rho_{\theta}(q)$ denote the probability that a seller of type $\theta$ draws $q \in\{g, b\}$, i.e., $\rho_{\theta}(g)=\theta=1-\rho_{\theta}(b)$. For any $\mathbf{h}^{k}=\left(q_{1}, \cdots, q_{k}\right) \in$ $H^{k}:=\{g, b\}^{k}$, let $\rho_{\theta}\left(\mathbf{h}^{k}\right)$ be the ex ante probability that $\mathbf{h}^{k}$ realizes for a seller of type $\theta$. We use $\mathbf{h}_{j}^{k}=\left(q_{1}, \cdots, q_{j}\right)$ to denote the first $j$-entry truncation of $\mathbf{h}^{k}$.

Given a default reputation $\mu_{0}>0$, let $\pi\left(\mathbf{h}_{j}^{k}\right)$ denote the posterior reputation for a seller who has survived the history $\mathbf{h}_{j}^{k}$ without cheating, updated according 
to $y_{v_{o}}^{\dagger}{ }^{18}$ Setting $\pi\left(\mathbf{h}_{0}^{k}\right)=\mu_{0}$, we can define $\pi\left(\mathbf{h}_{j}^{k}\right)$ recursively by:

$$
\pi\left(\mathbf{h}_{j}^{k}\right)=\frac{\pi\left(\mathbf{h}_{j-1}^{k}\right) \rho_{h}\left(q_{j}\right)}{\pi\left(\mathbf{h}_{j-1}^{k}\right) \rho_{h}\left(q_{j}\right)+\left(1-\pi\left(\mathbf{h}_{j-1}^{k}\right)\right) \rho_{\ell}\left(q_{j}\right)\left(1-y_{v_{o}}^{\dagger}\left(\pi\left(\mathbf{h}_{j-1}^{k}\right), h_{j}\right)\right)} .
$$

Then, the ex ante probability that an $\ell$-seller remains in the market without having cheated after $k$-period history $\mathbf{h}^{k}$ is

$$
\operatorname{Pr}\left(\mathbf{h}^{k}\right)=\prod_{j=1}^{k}\left[\rho_{\ell}\left(q_{j}\right)\left(1-y_{v_{o}}^{\dagger}\left(\pi\left(\mathbf{h}_{j-1}^{k}\right), q_{j}\right)\right)(1-\chi)\right]
$$

Consequently, in a stationary state, the measure of nominally $k$-period old $\ell$ sellers who restart in period $k+1$ for $k \geq 1$, is

$$
\chi_{0}\left(1-\mu_{0}\right)\left(\sum_{\mathbf{h}^{k} \in H^{k}} \operatorname{Pr}\left(\mathbf{h}^{k}\right)(1-\ell) y_{v_{o}}^{\dagger}\left(\pi\left(\mathbf{h}^{k}\right), b\right)(1-\chi)\right)
$$

where $\chi_{0}$ is a stationary-state measure of all sellers who start in each period. This implies that the total measure of old $\ell$-sellers who restart in an arbitrary period is $\chi_{0}\left(1-\mu_{0}\right) \Lambda\left(v_{o}\right)$ where

$$
\Lambda\left(v_{o}\right):=(1-\ell)(1-\chi) \sum_{k=1}^{\infty} \sum_{\mathbf{h}^{k} \in H^{k}} \operatorname{Pr}\left(\mathbf{h}^{k}\right) y_{v_{o}}^{\dagger}\left(\pi\left(\mathbf{h}^{k}\right), b\right) .
$$

Thus,

$$
\chi_{0}=\chi+\chi_{0}\left(1-\mu_{0}\right) \Lambda\left(v_{o}\right) \Longrightarrow \chi_{0}=\frac{\chi}{1-\left(1-\mu_{0}\right) \Lambda\left(v_{o}\right)} .
$$

Therefore, Bayes rule dictates that the following holds at a stationary state:

$$
\mu_{0}=\frac{\chi \mu_{i}}{\chi+\chi_{0}\left(1-\mu_{0}\right) \Lambda\left(v_{o}\right)}
$$

Solving (25) and (26) simultaneously, we define a mapping $\mu_{0}^{\dagger}:\left(\frac{\ell}{1-\delta}, \frac{1}{1-\delta}\right) \rightarrow$ $(0,1)$ as

$$
\mu_{0}^{\dagger}\left(v_{o}\right)=\frac{\mu_{i}-\mu_{i} \Lambda\left(v_{o}\right)}{1-\mu_{i} \Lambda\left(v_{o}\right)}<\mu_{i}
$$

where the inequality follows from $0<\Lambda\left(v_{o}\right)<1$.

The default value of $\ell$-seller in a stationary equilibrium is a fixed point $v_{o}$ that satisfies $V_{v_{o}}^{\dagger}\left(\mu_{0}^{\dagger}\left(v_{o}\right)\right)=v_{o}$. We show that such a fixed point exist by continuity, for which we need the next lemma.

\footnotetext{
${ }^{18}$ Recall $y_{v_{o}}^{\dagger}(\mu)=y_{v_{o}}^{\dagger}(\mu, b)$ and $y_{v_{o}}^{\dagger}(\mu, g)=0$.
} 
Lemma 9 Let $\psi:\left(\frac{\ell}{1-\delta}, \frac{1}{1-\delta}\right) \rightarrow \mathcal{C}_{[0,1]}$ be a mapping such that $\psi\left(v_{o}\right)=V_{v_{o}}^{\dagger}$ where $\mathcal{C}_{[0,1]}$ is the set of all continuous functions on $[0,1]$. Then, $\psi$ is continuous in $v_{o}$ under the sup norm at any $v_{o}>\frac{\ell}{1-\delta}$.

Proof. See Appendix.

Once existence of a default value $v_{o}$ is established, we obtain the equilibrium strategy $y_{v_{o}}^{\dagger}$ and value function $V_{v_{o}}^{\dagger}$ for $\ell$-seller as described in Section 5.1, and the default reputation level $\mu_{0}=\mu_{0}^{\dagger}\left(v_{o}\right)$ as per (27). Finally, by showing optimality of truth-telling for $h$-type, we obtain

Theorem 3 If sellers can change identity, a stationary NTR-equilibrium exists if $h(<1)$ and $\delta(<1)$ are sufficiently large.

Proof. Note that, as $v_{o} \rightarrow \frac{\ell}{1-\delta}, \mu_{0}^{\dagger}\left(v_{o}\right)$ converges to a limit strictly greater than 0 . Since the right derivative of $p_{G}\left(\mu, y_{v_{o}}^{\dagger}(\mu)\right)$ with respect to $\mu$ is uniformly bounded away from 0 at $\mu=0$, so is the right derivative of $V_{v_{o}}^{\dagger}(\mu)$ and consequently, $V_{v_{o}}^{\dagger}\left(\mu_{0}^{\dagger}\left(v_{o}\right)\right)>v_{o}$ for $v_{o}$ sufficiently close to $\frac{\ell}{1-\delta}$. On the other hand, as $v_{o} \rightarrow \frac{1}{1-\delta}$, since $\mu_{i}<1$ we have $V_{v_{o}}^{\dagger}\left(\mu_{0}^{\dagger}\left(v_{o}\right)\right) \leq V_{v_{o}}^{\dagger}\left(\mu_{i}\right)<V_{v_{o}}^{\dagger}(1) \leq v_{o}$ for $v_{o}$ sufficiently close to $\frac{1}{1-\delta}$. Then, since $\mu_{0}^{\dagger}\left(v_{o}\right)$ is continuous in $v_{o}$ from (27) and $\psi$ is continuous by Lemma 9, we must have $V_{v_{o}}^{\dagger}\left(\mu_{0}^{\dagger}\left(v_{o}\right)\right)=v_{o}$ for at least one $v_{o} \in\left(\frac{\ell}{1-\delta}, \frac{1}{1-\delta}\right)$.

Let $\mu_{0}^{s}$ and $v_{o}^{s}$ denote a pair of stationary default reputation level and value, i.e., $v_{o}^{s}=V_{v_{o}^{s}}^{\dagger}\left(\mu_{0}^{s}\right)$ and $\mu_{0}^{s}=\mu_{0}^{\dagger}\left(v_{o}^{s}\right)$. Note that to establish a stationary equilibrium, we still need to show that it is optimal for $h$-sellers to always report truthfully as long as $\mu \geq \mu_{0}^{s}$. Since the continuation value of $h$-seller after cheating is the equilibrium value of the default level $\mu_{0}^{s}, V_{h}^{\dagger}\left(\mu_{0}^{s}\right)$, rather than $V_{h}^{\dagger}(0)$, the optimality condition of $h$-seller is more difficult to verify than when restarting is impossible. In fact, it has not been proved that for all stationary pair of $\mu_{0}^{s}$ and $v_{o}^{s}$, truthful reporting for all $\mu \geq \mu_{0}^{s}$ is optimal for $h$-sellers when $\ell$-sellers report according to $y_{v_{o}^{s}}^{\dagger}(\mu)$ for $\mu \geq \mu_{0}^{s}$.

However, the proof of Lemma 1 relies on $V_{\ell}^{*}(0)$ being a constant, rather than $V_{\ell}^{*}(0)=\frac{\ell}{1-\delta}$ and consequently, applies analogously to $V_{h}^{\dagger}(\mu)$ defined as per $(47)$ in Appendix, with $y^{*}$ replaced by $y_{v_{o}^{s}}^{\dagger}$ for $\mu>\mu_{0}^{s}$. As a result, if $h>\frac{1+\sqrt{1+4 \ell^{2}+4 \ell^{3}}}{2+2 \ell}$, it constitutes an equilibrium for $\ell$-sellers to report according to $y_{v_{o}^{s}}^{\dagger}(\mu)$ and $h$ sellers honestly for $\mu \geq \mu_{0}^{s}$ for any stationary pair $\mu_{0}^{s}$ and $v_{o}^{s}$, provided that $\delta<1$ is sufficiently large so that, in particular, $\delta\left(V_{h}^{\dagger}(1)-V_{h}^{\dagger}\left(\mu_{0}^{s}\right)\right) \geq 1 .^{19}$ It may be worth mentioning that this is a sufficient condition, so stationary equilibria in which $h$-sellers behave honestly may exist in a wider class of environments.

\footnotetext{
${ }^{19}$ The proof is omitted because it is the same as the proof of Lemma 1 with obvious changes, such as $v_{o}^{s}$ and $\bar{\mu}^{\dagger}$ in place of $V_{\ell}^{*}(0)$ and $\bar{\mu}$, respectively.
} 
In any stationary NTR-equilibrium, since Proposition 2 applies without change, sellers' announcements are less reliable than when fresh restart with new identity is not possible.

However, this does not mean that untruthful announcements are more frequent in the market when restarts are possible than when they are not: $\ell$-sellers who have lied once, rather than keep lying forever when $q=b$, would start afresh and announce according to $y_{v_{o}}^{\dagger}(\mu)$. In fact, when $\delta$ is close to 1 there will be more truthful announcements in the market when sellers are allowed to restart with a new identity.

Nevertheless, $h$-sellers tend to suffer more due to untrustworthy behavior of $\ell$-sellers when restarts are possible, because such behavior by sellers who are "known" to be of $\ell$-type (i.e., those with reputation level $\mu=0$ ), which happens only when restarts are not allowed, does not affect $h$-sellers.

\section{Concluding Remarks}

In this paper we investigate the extent to which the quality of product can be credibly communicated to prospective buyers in experience good markets. We show that if there is adverse selection on seller's ability (in supplying good quality items), credible communication can be sustained by reputational motives in spite of the inherent conflict of interests between sellers and buyers. In addition, if sellers can restart with a new identity, a stationary equilibrium exists but the reliability of sellers' announcements deteriorates uniformly across all reputation levels.

To focus on the reputational incentives in pre-trade communication, we carried out our analysis in a model of pure adverse selection on seller's ability. However, the analysis can be extended to situations that involve moral hazard. To see this, modify the baseline model in such a way that in each period a seller draws an item of good quality with a probability $h$ if he exerted high effort at a cost of $c_{\theta}>0$ that depends on the seller's type $\theta \in\{h, \ell\}$, but he draws a good item with a probability $\ell$ if he exerted low effort at zero cost. Note that our NTR-equilibrium continues to be an equilibrium in this modified model if $c_{h}$ is small enough for an $h$-seller to find it worthwhile to exert high effort, but $c_{\ell}$ is large so that an $\ell$-seller finds otherwise. ${ }^{20}$ If pre-trade communication

\footnotetext{
${ }^{20}$ This is the case if $\delta\left(V_{h}^{*}\left(\pi_{B b}\left(\mu, y^{*}(\mu)\right)-\frac{c_{h}}{1-\delta}-V_{h}^{*}(0)\right) \geq p_{G}\left(\mu, y^{*}(\mu)\right)\right.$ for all $\mu$, and the inequality is reversed if $c_{\ell}$ replaces $c_{h}$, where $V_{h}^{*}(0)=\frac{\ell}{1-\delta}=V_{\ell}^{*}(0)$ and $y^{*}, V_{\ell}^{*}$ and $V_{h}^{*}$ are as derived in Section 3. Such values of $c_{h}$ and $c_{\ell}$ exist because $\left.i\right) \delta\left(V_{h}^{*}\left(\pi_{B b}\left(\mu, y^{*}(\mu)\right)-V_{h}^{*}(0)\right)>\right.$ $p_{G}\left(\mu, y^{*}(\mu)\right)$ for $\mu \geq \bar{\mu}$ if $\delta>\delta_{h}$ due to $(15)$, ii) $\delta\left(V_{\ell}^{*}\left(\pi_{B b}\left(\mu, y^{*}(\mu)\right)-V_{\ell}^{*}(0)\right)=p_{G}\left(\mu, y^{*}(\mu)\right)\right.$ by definition of $V_{\ell}^{*}$, and iii) $V_{h}^{*}\left(\pi_{B b}\left(\mu, y^{*}(\mu)\right)>V_{\ell}^{*}\left(\pi_{B b}\left(\mu, y^{*}(\mu)\right)+\zeta\right.\right.$ for some $\zeta>0$ due to Lemma 8, continuity of $V_{\theta}^{*}$ for $\mu>0$, and $\lim _{\mu \rightarrow 0} \pi_{B b}\left(\mu, y^{*}(\mu)\right)>0$, where the last inequality is implied by $\lim _{\mu \rightarrow 0} \delta\left(V_{\ell}^{*}\left(\pi_{B b}\left(\mu, y^{*}(\mu)\right)-V_{\ell}^{*}(0)\right)=\ell\right.$.
} 
is not possible, this model is equivalent to the baseline model of Mailath and Samuelson (2001) without replacement of types, for which they show that high effort cannot be induced unless discontinuous strategies are allowed (Proposition 2 , p424). Our result suggests that pre-trade communication may motivate the more efficient type to exert high effort by facilitating the learning process in the market.

We anticipate that our analysis can be extended in other directions as well. For instance, in the context of internet markets, to examine the effect of competition between trading websites appears as an interesting task from the market design perspective. Analysis of such competition may also carry implications on the market segmentation between trading websites and their pricing strategies. We intend to address these issues in the future.

\section{APPENDIX}

Proof of Lemma 3. (i) If $y^{*}(1)<1$, we would have

$$
V_{\ell}^{*}(1)=\ell\left(1+\delta V_{\ell}^{*}(1)\right)+(1-\ell)\left(y^{*}(1)\left(1+\delta V_{\ell}^{*}\left(\pi_{G b}^{*}(1)\right)+\left(1-y^{*}(1)\right) \delta V_{\ell}^{*}(1)\right)\right.
$$

along with $1+\delta V_{\ell}^{*}\left(\pi_{G b}^{*}(1)\right) \leq \delta V_{\ell}^{*}\left(\pi_{B b}^{*}(1)\right)=\delta V_{\ell}^{*}(1)$, whence

$$
\begin{gathered}
V_{\ell}^{*}(1) \leq \ell\left(1+\delta V_{\ell}^{*}(1)\right)+(1-\ell) \delta V_{\ell}^{*}(1)=\ell+\delta V_{\ell}^{*}(1) \\
\Longrightarrow \quad V_{\ell}^{*}(1) \leq \ell /(1-\delta)=V_{\ell}^{*}(0) \Longrightarrow V_{\ell}^{*}(1)=V_{\ell}^{*}(0),
\end{gathered}
$$

i.e., $V_{\ell}^{*}$ would be constant. Since this would contradict the earlier asserted inequality $1+\delta V_{\ell}^{*}\left(\pi_{G b}^{*}(1)\right) \leq \delta V_{\ell}^{*}\left(\pi_{B b}^{*}(1)\right)$, we conclude that $y^{*}(1)=1$.

Next, suppose that $\lim _{\mu \rightarrow 1} y^{*}(\mu) \neq 1$. Then, the following holds for some $\eta>0$ : for any $\epsilon>0$ there is $\mu_{\epsilon}<1$ such that $1-\epsilon<\mu_{\epsilon}$ and $y^{*}\left(\mu_{\epsilon}\right)<1-\eta$ and thus,

$$
V_{\ell}^{*}\left(\mu_{\epsilon}\right)=\ell p_{G}\left(\mu_{\epsilon}, y^{*}\left(\mu_{\epsilon}\right)\right)+\delta\left(\ell V_{\ell}^{*}\left(\pi_{G g}\left(\mu_{\epsilon}\right)\right)+(1-\ell) V_{\ell}^{*}\left(\pi_{B b}\left(\mu_{\epsilon}, y^{*}\left(\mu_{\epsilon}\right)\right)\right)\right) .
$$

Since $p_{G}\left(\mu_{\epsilon}, y^{*}\left(\mu_{\epsilon}\right)\right) \rightarrow 1, \mu_{\epsilon} \rightarrow 1, \pi_{G g}\left(\mu_{\epsilon}\right) \rightarrow 1$, and $\pi_{B b}\left(\mu_{\epsilon}, y^{*}\left(\mu_{\epsilon}\right)\right) \rightarrow 1$ as $\epsilon \rightarrow 0$, (28) would imply $\lim _{\mu \rightarrow 1} V_{\ell}^{*}(\mu)=\ell+\delta \lim _{\mu \rightarrow 1} V_{\ell}^{*}(\mu)$, i.e., $\lim _{\mu \rightarrow 1} V_{\ell}^{*}(\mu)=\frac{\ell}{1-\delta}=$ $V_{\ell}^{*}(0)$. Then, $V_{\ell}^{*}(\mu)=V_{\ell}^{*}(0)$ for all $\mu$ which would imply that an $\ell$-seller with a bad quality product would always lie as $p_{G}\left(\mu_{\epsilon}, y^{*}\left(\mu_{\epsilon}\right)\right)+\delta V_{\ell}^{*}(0)>\delta V_{\ell}^{*}(0)$, contradicting $y^{*}\left(\mu_{\epsilon}\right)<1$. Hence, we conclude that $\lim _{\mu \rightarrow 1} y^{*}(\mu)=1$.

(ii) Since $y^{*}(\mu)>0$ by (i) for all sufficiently large $\mu<1$, we have $V_{\ell}^{*}(\mu)=$ $p_{G}\left(\mu, y^{*}(\mu)\right)+\delta\left(\ell V_{\ell}^{*}\left(\pi_{G g}(\mu)\right)+(1-\ell) V_{\ell}^{*}(0)\right)$ as $\mu \rightarrow 1$, and thus,

$$
\lim _{\mu \rightarrow 1} V_{\ell}^{*}(\mu)=\frac{p_{G}(1,1)+\delta(1-\ell) V_{\ell}^{*}(0)}{1-\delta \ell}=\frac{1-\delta\left(1-\ell+\ell^{2}\right)}{(1-\delta)(1-\delta \ell)} .
$$


(iii) With a view to reach a contradiction, suppose that $y^{*}(\mu)<1$ for some $\mu$, yet $\bar{V}_{\ell}<V_{\ell}^{*}(1)$. Note that $\delta\left(\bar{V}_{\ell}-V_{\ell}^{*}(0)\right)<1$ from $(6)$.

If $\delta\left(V_{\ell}^{*}(1)-V_{\ell}^{*}(0)\right)>\ell$, then there is some $\mu \in(0,1)$ such that $\delta\left(V_{\ell}^{*}(1)-V_{\ell}^{*}(0)\right)>$ $p_{G}(\mu, 1)>\delta\left(\bar{V}_{\ell}-V_{\ell}^{*}(0)\right)$ so that $y^{*}(\mu)$ cannot be equal to 1 because $\delta\left(V_{\ell}^{*}(1)-V_{\ell}^{*}(0)\right)>$ $p_{G}(\mu, 1)$, nor can it be less than 1 because $\delta\left(V_{\ell}^{*}\left(\pi_{B b}(\mu, y)\right)-V_{\ell}^{*}(0)\right)<p_{G}(\mu, y)$ for all $y<1$, which is impossible.

If $\delta\left(V_{\ell}^{*}(1)-V_{\ell}^{*}(0)\right) \leq \ell$, on the other hand, $y^{*}(\mu)=1$ must hold for all $\mu \in(0,1]$ due to condition (ii) of Definition 1 , because $p_{G}(\mu, y)>\ell \geq \delta\left(V_{\ell}^{*}(1)-V_{\ell}^{*}(0)\right) \geq$ $\delta\left(V^{*}\left(\pi_{B b}(\mu, y)\right)-V^{*}\left(\pi_{G b}(\mu, y)\right)\right)$ for any $y$. Since $y^{*}(0)=1$ as asserted in (2), we have encountered a contradiction to the supposition that $y^{*}(\mu)<1$ for some $\mu$.

Therefore, we conclude that $\bar{V}_{\ell}=V_{\ell}^{*}(1)$. This, together with $V_{\ell}^{*}(1)=1+$ $\delta\left(\ell V_{\ell}^{*}(1)+(1-\ell) V_{\ell}^{*}\left(\pi_{G b}^{*}(1)\right)\right.$, implies that $V_{\ell}^{*}\left(\pi_{G b}^{*}(1)\right)=V_{\ell}^{*}(0)$.

(iv) Suppose we reset $\pi_{G b}^{*}(1)=0$ in an equilibrium with $y^{*}(\mu) \equiv 1$. The value function $V_{h}^{*}($.$) is unchanged, while the incentive compatibility condition for type h$ at 1 is still verified: $1+\delta V_{h}^{*}(1) \geq \delta V_{h}^{*}(0)$ holds if it holds for $\pi_{G b}^{*}(1)>0$. For the $\ell$-type seller, the value $V_{\ell}^{*}(1)$ is lower while $V_{\ell}^{*}(\mu)$ is unchanged for $\mu<1$. But since $\mu_{t}=1$ should never occur in equilibrium when the type is $\ell$, all incentive compatibility are preserved. Hence, it still constitutes an equilibrium when $\pi_{G b}^{*}(1)$ is reset at 0 . It is trivial to verify that $\bar{V}_{\ell}=V_{\ell}^{*}(1)$ holds in this equilibrium.

Proof of Lemma 7. To reach a contradiction, suppose that there exists $\mu$ such that $\pi_{B b}\left(\mu, y_{V}(\mu)\right) \leq \mu$. Since $y_{V}$ is continuous (Lemma 4) and $y_{V}(\mu)=1$ for $\mu \geq \bar{\mu}$, there exists

$$
\tilde{\mu}=\max \left\{\mu<1 \mid \pi_{B b}\left(\mu, y_{V}(\mu)\right) \leq \mu\right\}<\bar{\mu} .
$$

Note that $\pi_{B b}\left(\tilde{\mu}, y_{V}(\tilde{\mu})\right)=\tilde{\mu}$. Since it is easily verified from (5) that

$$
\pi_{B b}(\mu, y) \geq \mu \Longleftrightarrow y \geq \hat{y}:=\frac{h-\ell}{1-\ell},
$$

it must be the case that $y_{V}(\tilde{\mu})=\hat{y}$ and thus

$$
p_{G}(\tilde{\mu}, \hat{y})=\delta(V(\tilde{\mu})-V(0)) .
$$

Expanding $V(\mu)=p_{G}\left(\mu, y^{*}(\mu)\right)+\delta\left(\ell V\left(\pi_{G g}(\mu)\right)+(1-\ell) V(0)\right)$ by applying an analogous equation to $V\left(\pi_{G g}(\mu)\right)$ repeatedly, we get

$$
\begin{aligned}
V(\mu) & =\left[\sum_{t=0}^{\infty} \ell^{t} \delta^{t} p_{G}\left(\pi_{G g}^{t}(\mu), y_{V}\left(\pi_{G g}^{t}(\mu)\right)\right)\right]+\delta V(0)(1-\ell) \sum_{t=0}^{\infty} \ell^{t} \delta^{t} \\
& =\sum_{t=0}^{\infty} \delta^{t} \ell^{t}\left(p_{G}\left(\pi_{G g}^{t}(\mu), y_{V}\left(\pi_{G g}^{t}(\mu)\right)\right)-\ell\right)+V(0) .
\end{aligned}
$$

where $\pi_{G g}^{t}(\mu)=\pi_{G g}\left(\pi_{G g}^{t-1}\right)$ is defined recursively so that

$$
\pi_{G g}^{t}(\mu)=\frac{\mu h^{t}}{\mu h^{t}+(1-\mu) \ell^{t}} .
$$


Note from (34) that $\pi_{G g}^{t}(\tilde{\mu})>\tilde{\mu}$ for $t>0$ and thus, $\pi_{B b}\left(\pi_{G g}^{t}(\tilde{\mu}), y_{V}\left(\pi_{G g}^{t}(\tilde{\mu})\right)\right)>$ $\pi_{G g}^{t}(\tilde{\mu})$ by (29). Consequently, $y_{V}\left(\pi_{G g}^{t}(\tilde{\mu})\right)>\hat{y}$ by $(30)$. Therefore, since $p_{G}(\mu, y) \leq 1$ and $p_{G}(\mu, y)$ decreases in $y,(33)$ implies that

$$
V(\tilde{\mu})-V(0)<\sum_{t=0}^{\infty}\left(p_{G}\left(\pi_{G g}^{t}(\tilde{\mu}), \hat{y}\right)-\ell\right) \delta^{t} \ell^{t} .
$$

Since

$$
p_{G}(\mu, \hat{y})=\frac{\mu h+(1-\mu) \ell}{h}
$$

from (3), we further deduce from (35) that

$$
\begin{aligned}
V(\tilde{\mu})-V(0) & <p_{G}(\tilde{\mu}, \hat{y})-\ell+\sum_{t=1}^{\infty}\left(\frac{\pi_{G g}^{t}(\tilde{\mu})(h-\ell)+\ell(1-h)}{h}\right) \delta^{t} \ell^{t} \\
& <p_{G}(\tilde{\mu}, \hat{y})-\ell+\sum_{t=1}^{\infty}\left(\frac{(h-\ell)+\ell(1-h)}{h}\right) \delta^{t} \ell^{t} \\
& =p_{G}(\tilde{\mu}, \hat{y})-\ell+(1-\ell) \frac{\delta \ell}{1-\delta \ell} \\
& =p_{G}(\tilde{\mu}, \hat{y})-\frac{(1-\delta) \ell}{1-\delta \ell}<p_{G}(\tilde{\mu}, \hat{y})
\end{aligned}
$$

where the second inequality follows from $\pi_{G g}^{t}(\tilde{\mu})<1$. Thus, we have reached a contradictory conclusion that (31) cannot hold at $\tilde{\mu}$.

Proof of Theorem 1. Proof of existence. Define $\mathcal{F}^{r}$ as the subset of all rightcontinuous functions in $\mathcal{F}$, endowed with the topology of the weak convergence. The set $\mathcal{F}^{r}$ is convex and compact (Theorem 5.1, Billingsley, 1999). By Fan-Glicksberg Fixed Point Theorem, ${ }^{21}$ therefore, $T$ has a fixed point in $\mathcal{F}^{r}$ if

$$
T\left(\mathcal{F}^{r}\right) \subset \mathcal{F}^{r} \text { and } T \text { is continuous on } \mathcal{F}^{r} .
$$

We now show (37). First note that $T(V)$ is right-continuous if $V$ is rightcontinuous because $y_{V}(\mu)$ is continuous in $\mu$, which proves $T\left(\mathcal{F}^{r}\right) \subset \mathcal{F}^{r}$.

Next, consider a sequence $V_{n}, n=1,2, \cdots$, in $\mathcal{F}^{r}$ that weakly converges to $V \in$ $\mathcal{F}^{r}$. To prove continuity of $T$, we show below that $T\left(V_{n}\right)$ weakly converges to $T(V)$, i.e., $T\left(V_{n}\right)(\mu)$ converges to $T(V)(\mu)$ at all continuity points of $T(V)$ (Theorem 2.1, Billingsley, 1999).

Let $\Omega$ be the set of all points where $V\left(\pi_{G g}(\mu)\right)$ is continuous. Since $\pi_{G g}(\mu)$ is

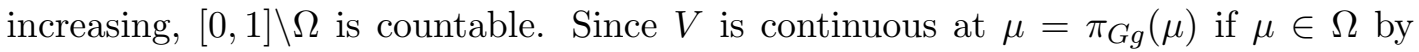
continuity of $\pi_{G g}$, it follows that $V_{n}\left(\pi_{G g}(\mu)\right)$ converges to $V\left(\pi_{G g}(\mu)\right)$ on $\Omega$.

\footnotetext{
${ }^{21}$ This theorem (Fan, 1952; Glicksberg, 1952) states that an upper hemi-continuous convex valued correspondence from a nonempty compact convex subset of a convex Hausdorff topological vector space has a fixed point.
} 
Next, Let $y_{V}(\mu)$ be as defined in (11) for $V$ and $y_{V_{n}}(\mu)$ for $V_{n}$. Let $\Lambda$ be the set of points where $V\left(\pi_{B b}\left(\mu, y_{V}(\mu)\right)\right)$ is continuous. Since $\pi_{B b}\left(\mu, y_{V}(\mu)\right)$ is non-decreasing on $(0,1]$ as verified in the proof of Lemma $5,[0,1] \backslash \Lambda$ is countable. We now show that $y_{V_{n}}(\mu) \rightarrow y_{V}(\mu)$ for all $\mu \in \Lambda$.

Consider $\mu \in \Lambda$. That $y_{V_{n}}(\mu) \rightarrow y_{V}(\mu)$ is trivial from (11) if $\mu=0$ or $\mu>\bar{\mu}$. Hence, suppose $0<\mu \leq \bar{\mu}$ so that, with $V_{n}^{-}$denoting the left limit,

$$
\delta\left(V_{n}^{-}\left(\pi_{B b}\left(\mu, y_{V_{n}}(\mu)\right)\right)-V(0)\right) \leq p_{G}\left(\mu, y_{V_{n}}(\mu)\right) \leq \delta\left(V\left(\pi_{B b}\left(\mu, y_{V_{n}}(\mu)\right)\right)-V(0)\right) .
$$

By taking a subsequence if necessary, we may assume that $y_{V_{n}}(\mu)$ converges to a limit $y^{\prime}$. To reach a contradiction, suppose $y^{\prime} \neq y_{V}(\mu)$. First, consider the case that $y^{\prime}<y_{V}(\mu)$. Then, since $p_{G}(\mu, y)$ decreases with $\mu$ there exists $\varepsilon>0$ such that

$$
p_{G}\left(\mu, y_{V_{n}}(\mu)\right)>p_{G}\left(\mu, y_{V}(\mu)\right)+\varepsilon=\delta\left(V\left(\pi_{B b}\left(\mu, y_{V}(\mu)\right)\right)-V(0)\right)+\varepsilon
$$

for sufficiently large $n$, where the equality follows because $\mu \in \Lambda$. From this we further deduce that

$$
\begin{aligned}
p_{G}\left(\mu, y_{V_{n}}(\mu)\right) & >\delta\left(V_{n}\left(\pi_{B b}\left(\mu, y_{V}(\mu)\right)\right)-V(0)\right)+\varepsilon / 2 \\
& >\delta\left(V_{n}\left(\pi_{B b}\left(\mu, y_{V_{n}}(\mu)\right)\right)-V(0)\right)+\varepsilon / 2
\end{aligned}
$$

for sufficiently large $n$, where the first inequality follows because $V_{n}\left(\pi_{B b}\left(\mu, y_{V}(\mu)\right)\right) \rightarrow$ $V\left(\pi_{B b}\left(\mu, y_{V}(\mu)\right)\right)$ for $\mu \in \Lambda$ and the second because $\pi_{B b}(\mu, y)$ increases in $y$ and $y_{V_{n}}(\mu) \rightarrow y^{\prime}<y_{V}(\mu)$. However, this contradicts (38).

For the case $y^{\prime}>y_{V}(\mu)$, we can apply the same reasoning using $V_{n}^{-}\left(\pi_{B b}\left(\mu, y_{V_{n}}(\mu)\right)\right) \geq$ $V_{n}\left(\pi_{B b}\left(\mu, y_{V}(\mu)\right)\right)$ for $n$ large to reach an analogous contradiction:

$$
p_{G}\left(\mu, y_{V_{n}}(\mu)\right)<\delta\left(V_{n}^{-}\left(\pi_{B b}\left(\mu, y_{V_{n}}(\mu)\right)\right)-V(0)\right)-\varepsilon / 2 .
$$

Hence, we conclude that $y_{V_{n}}(\mu) \rightarrow y_{V}(\mu)$ for all $\mu \in \Lambda$.

Together with the earlier result that $V_{n}\left(\pi_{G g}(\mu)\right) \rightarrow V\left(\pi_{G g}(\mu)\right)$ for all $\mu \in \Omega$, this establishes for all $\mu \in \Omega \cap \Lambda$ that

$$
\begin{aligned}
T\left(V_{n}\right)(\mu) & =p_{G}\left(\mu, y_{V_{n}}(\mu)\right)+\delta\left(\ell V_{n}\left(\pi_{G g}(\mu)\right)+(1-\ell) V(0)\right) \\
& \rightarrow p_{G}\left(\mu, y_{V}(\mu)\right)+\delta\left(\ell V\left(\pi_{G g}(\mu)\right)+(1-\ell) V(0)\right)=T(V)(\mu)
\end{aligned}
$$

as $n \rightarrow \infty$. Finally, to verify this convergence at every continuity point of $T(V)(\mu)$, observe first that this convergence is trivial from (11) at $\mu=0,1$. For any other $\mu \notin \Omega \cap \Lambda$ at which $T(V)$ is continuous, one can find $\mu_{1} \in \Omega \cap \Lambda \cap(0, \mu)$ arbitrarily close to $\mu$ and $\mu_{2} \in \Omega \cap \Lambda \cap(\mu, 1)$ arbitrarily close to $\mu$ because $\Omega \cap \Lambda$ is dense in $[0,1]$. Since $T\left(V_{n}\right)\left(\mu_{1}\right) \leq T\left(V_{n}\right)(\mu) \leq T\left(V_{n}\right)\left(\mu_{2}\right)$ and $T(V)\left(\mu_{1}\right) \leq T(V)(\mu) \leq T(V)\left(\mu_{2}\right)$, taking the limits we get

$$
\begin{gathered}
T(V)\left(\mu_{1}\right) \leq \liminf T\left(V_{n}\right)(\mu) \leq \lim \sup T\left(V_{n}\right)(\mu) \leq T(V)\left(\mu_{2}\right), \quad \text { and } \\
\sup _{\substack{\mu_{1} \in \Omega \cap \Lambda \\
\mu_{1}<\mu}} T(V)\left(\mu_{1}\right)=T(V)(\mu)=\inf _{\substack{\mu_{2} \in \Omega \cap \Lambda \\
\mu_{2}>\mu}} T(V)\left(\mu_{2}\right),
\end{gathered}
$$


which imply, as desired, that $T\left(V_{n}\right)(\mu)$ converges to $T(V)(\mu)$ at every continuity point of $T(V)(\mu)$. This proves (37), thus completes the proof of existence.

Proof of uniqueness. To reach a contradiction, suppose there are two fixed points $V^{1}$ and $V^{2}$. Notice that the level $\bar{\mu}$ is independent of $V$ and

$$
V^{i}(\mu)=p_{G}(\mu, 1)+\delta(\ell V(1)+(1-\ell) V(0)) \quad \forall \mu \geq \bar{\mu} .
$$

Since $V^{1}(\mu)=V^{2}(\mu)$ for all $\mu \geq \bar{\mu}$, the following is well-defined:

$$
\hat{\mu}:=\min \left\{\mu \mid V^{1}\left(\mu^{\prime}\right)=V^{2}\left(\mu^{\prime}\right) \forall \mu^{\prime} \geq \mu\right\} \in(0, \bar{\mu}] .
$$

A "segment" for $i=1,2$, is a nonempty interval $I_{i}=[x, z] \subset[0, \bar{\mu}]$ such that $V^{i}(\mu)>$ $V^{j}(\mu)$ for all $\mu \in(x, z)$ and $V^{i}(\mu)=V^{j}(\mu)$ for $\mu=x, z$, where $j \neq i$. A "region" for $i=1,2$, is a nonempty interval $R_{i}=[x, z] \subset[0, \bar{\mu}]$ such that $V^{i}(\mu) \geq V^{j}(\mu)$ for all $\mu \in I_{i}$ and there are $x^{\prime}, z^{\prime} \in R_{i}$ such that $\left[x, x^{\prime}\right]$ and $\left[z^{\prime}, z\right]$ are segments for $i$. Let

$$
p_{G}^{i}(\mu):=p_{G}\left(\mu, y_{V^{i}}(\mu)\right) \quad \text { and } \quad \pi_{B b}^{i}(\mu):=\pi_{B b}\left(\mu, y_{V^{i}}(\mu)\right) \quad \text { for } \quad i=1,2 .
$$

Recall that in the proof of Lemma 5, we have shown that both $p_{G}^{i}(\mu)$ and $\pi_{B b}^{i}(\mu)$ weakly increase in $\mu$. Since $V^{i}$ strictly increases in $\mu$ by Lemma 6 , the same reasoning establishes that

[A] $p_{G}^{i}(\mu)$ and $\pi_{B b}^{i}(\mu)$ strictly increase in $\mu$.

Next, we establish the following:

[B] If $V^{1}\left(\pi_{B b}^{i}(\mu)\right)=V^{2}\left(\pi_{B b}^{i}(\mu)\right)$ for some $\mu>0$ and some $i=1,2$, then $y^{1}(\mu)=$ $y^{2}(\mu)$ and consequently, $p_{G}^{1}(\mu)=p_{G}^{2}(\mu)$ and $\pi_{B b}^{1}(\mu)=\pi_{B b}^{2}(\mu)$. If, in addition, $V^{1}\left(\pi_{G g}(\mu)\right)=V^{2}\left(\pi_{G g}(\mu)\right)$ holds, then $V^{1}(\mu)=V^{2}(\mu)$.

Note that this observation is trivial for $\mu \geq \bar{\mu}$. Since

$$
p_{G}^{i}(\mu)=\delta\left(V^{i}\left(\pi_{B b}^{i}(\mu)\right)-V^{i}(0)\right) \quad \forall \mu \in(0, \bar{\mu}],
$$

$V^{1}\left(\pi_{B b}^{i}(\mu)\right)=V^{2}\left(\pi_{B b}^{i}(\mu)\right)$ implies $p_{G}^{1}(\mu)=p_{G}^{2}(\mu)$, which in turn implies $y_{V_{\ell}^{1}}^{*}(\mu)=$ $y_{V_{\ell}^{2}}^{*}(\mu)$, from which the remaining claims of $[\mathrm{B}]$ follow.

Finally, since $\pi_{B b}^{i}(\hat{\mu})>\hat{\mu}$ by Lemma 7 and $\pi_{G g}(\hat{\mu})>\hat{\mu}$ by (4), due to continuity, there is $\mu^{\prime}<\hat{\mu}$ such that $V^{1}\left(\mu^{\prime}\right) \neq V^{2}\left(\mu^{\prime}\right), \pi_{B b}^{i}\left(\mu^{\prime}\right)>\hat{\mu}$ and $\pi_{G g}\left(\mu^{\prime}\right)>\hat{\mu}$. Then, $V^{1}\left(\pi_{B b}^{i}\left(\mu^{\prime}\right)\right)=V^{2}\left(\pi_{B b}^{i}\left(\mu^{\prime}\right)\right)$ by (40) and thus, $V^{1}\left(\mu^{\prime}\right)=V^{2}\left(\mu^{\prime}\right)$ by [B], a contradiction to the earlier assertion that $V^{1}\left(\mu^{\prime}\right) \neq V^{2}\left(\mu^{\prime}\right)$. This completes the proof of uniqueness, hence of Theorem 1.

Proof of Lemma 8. Let $V_{h}(\mu)$ be the value function from the following strategy of an $h$-seller: always report $q=g$ truthfully and upon drawing $q=b$ for the first time report $m=G$ and get $V_{h}^{*}(0)$ in the continuation subgame. Then,

$$
V_{h}(\mu)=\left[\sum_{t=0}^{\infty} h^{t} \delta^{t} p_{G}\left(\pi_{G g}^{t}(\mu), y^{*}\left(\pi_{G g}^{t}(\mu)\right)\right)\right]+\delta V_{h}^{*}(0)(1-h) \sum_{t=1}^{\infty} h^{t} \delta^{t}
$$


where $\pi_{G g}^{t}(\mu)=\pi_{G g}\left(\pi_{G g}^{t-1}(\mu)\right)$ as defined in (34). Clearly, $V_{h}^{*}(\mu) \geq V_{h}(\mu)$.

From equation (33) in the proof of lemma 7 ,

$$
V_{\ell}^{*}(\mu)=\left[\sum_{t=0}^{\infty} \ell^{t} \delta^{t} p_{G}\left(\pi_{G g}^{t}(\mu), y^{*}\left(\pi_{G g}^{t}(\mu)\right)\right)\right]+\delta V_{\ell}^{*}(0)(1-\ell) \sum_{t=0}^{\infty} \ell^{t} \delta^{t} .
$$

Subtracting (44) from (43),

$$
\begin{aligned}
V_{h}(\mu)-V_{\ell}^{*}(\mu)= & {\left[\sum_{t=0}^{\infty}\left(h^{t}-\ell^{t}\right) \delta^{t} p_{G}\left(\pi_{G g}^{t}(\mu), y^{*}\left(\pi_{G g}^{t}(\mu)\right)\right)\right] } \\
& +\delta\left(\frac{1-h}{1-\delta h}-\frac{1-\ell}{1-\delta \ell}\right) V_{\ell}^{*}(0) .
\end{aligned}
$$

Using $p_{G}\left(\pi_{G g}^{t}(\mu), y^{*}\left(\pi_{G g}^{t}(\mu)\right)\right)>\ell$, we have

$$
V_{h}(\mu)-V_{\ell}^{*}(\mu)>\frac{\delta(h-\ell) \ell}{(1-\delta h)(1-\delta \ell)}-\frac{\delta(1-\delta)(h-\ell)}{(1-\delta h)(1-\delta \ell)} V_{\ell}^{*}(0)=0 .
$$

Proof of Theorem 2. By construction the incentive compatibility conditions are satisfied for the $\ell$-seller at the unique fixed point of $T$. Moreover, with $V_{h}^{*}(0)=$ $\ell /(1-\delta)$ the optimal strategy for an $h$-seller is to announce $m=q$ if $q=g$. It suffices to show that $x^{*}(\mu, b)=0$ for $\mu>0$.

For $\mu \in[\bar{\mu}, 1]$, this follows from (15) because $\pi_{B b}\left(\mu, y^{*}(\mu)\right)=1$ and $p_{G}\left(\mu, y^{*}(\mu)\right) \leq$ 1. For $\mu \in(0, \bar{\mu})$, observe from (10) and (11) that

$$
\delta\left(V_{\ell}^{*}\left(\pi_{B b}\left(\mu, y^{*}(\mu)\right)\right)-V_{\ell}^{*}(0)\right)=p_{G}\left(\mu, y^{*}(\mu)\right) .
$$

Since $V_{h}^{*}\left(\pi_{B b}\left(\mu, y^{*}(\mu)\right)\right)>V_{\ell}^{*}\left(\pi_{B b}\left(\mu, y^{*}(\mu)\right)\right)$ by Lemma 8 while $V_{\ell}^{*}(0)=V_{h}^{*}(0)$,

$$
\delta\left(V_{h}^{*}\left(\pi_{B b}\left(\mu, y^{*}(\mu)\right)\right)-V_{h}^{*}(0)\right)>p_{G}\left(\mu, y^{*}(\mu)\right) .
$$

Hence, we have proved the optimality of $x^{*}(\mu, b)=0$ for $\mu>0$.

We already showed that $x^{*}(0, b)=1$ is optimal with $\pi_{B b}^{*}(0)=0$, which completes description of an NTR-equilibrium. Other NTR-equilibria may exist that differ in $x^{*}(0, b)$ and $\pi_{B b}^{*}(0)$. But since consistency requires that an $h$-seller starts with an initial reputation level $\mu>0$ and an $h$-seller always tells the truth as per property (a) of NTR-equilibrium, specification of $x^{*}(0, b)$ is a part of off-equilibrium strategy. Therefore, the equilibrium outcome is unique.

Finally, the value function for the $h$-type is given by

$$
V_{h}^{*}(\mu)=\sum_{t=0}^{\infty} \sum_{\mathbf{h}^{t} \in H_{g}^{t}} \delta^{t} \rho\left(\mathbf{h}^{t}\right) p_{G}\left(\pi\left(\mathbf{h}^{t}, \mu\right), y^{*}\left(\pi\left(\mathbf{h}^{t}, \mu\right)\right)\right)
$$

where $H_{g}^{t}:=\{g, b\}^{t-1} \times\{g\}$ is the set of all possible realizations of $q$ for $t$ periods with the requirement that $q=g$ in period $t ; \rho\left(\mathbf{h}^{t}\right)$ is the ex ante probability that 
$\mathbf{h}^{t} \in H_{g}^{t}$ realizes; $\pi\left(\mathbf{h}^{t}, \mu\right)$ is the posterior belief at the beginning of period $t$ calculated by Bayes rule from the prior belief $\mu$ along $\mathbf{h}^{t}$. Observe that $V_{h}^{*}(\mu)$ is increasing in $\mu$ because $p_{G}\left(\mu, y^{*}(\mu)\right), \pi_{G g}(\mu)$ and $\pi_{B b}\left(\mu, y^{*}(\mu)\right)$ all increase in $\mu$ as verified earlier.

Proof of Proposition 1. Let

$$
V_{h}^{o}(\mu):=h \sum_{t=0}^{\infty} h^{t} \delta^{t} p_{G}\left(\pi_{G g}^{t}(\mu), y^{*}\left(\pi_{G g}^{t}(\mu)\right)\right) \quad \forall \mu>0
$$

so that

$$
V_{h}^{*}(\mu)=V_{h}^{o}(\mu)+(1-h) \delta \sum_{t=0}^{\infty} h^{t} \delta^{t} V_{h}^{*}\left(\pi_{B b}\left(\pi_{G g}^{t}(\mu), y^{*}\left(\pi_{G g}^{t}(\mu)\right)\right) \quad \forall \mu>0 .\right.
$$

In conjunction with (32), we have

$$
V_{h}^{o}(\mu)-V_{\ell}^{*}(\mu)=\left[\sum_{t=0}^{\infty}\left(h^{t+1}-\ell^{t}\right) \delta^{t} p_{G}\left(\pi_{G g}^{t}(\mu), y^{*}\left(\pi_{G g}^{t}(\mu)\right)\right)\right]-\delta V_{\ell}^{*}(0) \frac{1-\ell}{1-\delta \ell}
$$

and thus,

$$
\begin{gathered}
\frac{d V_{h}^{o}(\mu)}{d \mu}-\frac{d V_{\ell}^{*}(\mu)}{d \mu}=\sum_{t=0}^{\infty}\left(h^{t+1}-\ell^{t}\right) \delta^{t} \frac{\partial p_{G}\left(\pi_{G g}^{t}(\mu), 1\right)}{\partial \mu} \frac{d \pi_{G g}^{t}(\mu)}{d \mu} \\
=\sum_{t=0}^{\infty} \delta^{2 t}\left[\left(h^{2 t+1}-\ell^{2 t}\right) \frac{\partial p_{G}\left(\pi_{G g}^{2 t}(\mu), 1\right)}{\partial \mu} \frac{d \pi_{G g}^{2 t}(\mu)}{d \mu}\right. \\
\left.\quad+\delta\left(h^{2 t+2}-\ell^{2 t+1}\right) \frac{\partial p_{G}\left(\pi_{G g}^{2 t+1}(\mu), 1\right)}{\partial \mu} \frac{d \pi_{G g}^{2 t+1}(\mu)}{d \mu}\right] \\
>\sum_{t=0}^{\infty} \delta^{2 t} \ell^{2 t}\left[(h-1) \frac{\partial p_{G}\left(\pi_{G g}^{2 t}(\mu), 1\right)}{\partial \mu} \frac{d \pi_{G g}^{2 t}(\mu)}{d \mu}\right. \\
\left.+\delta\left(h^{2}-\ell\right) \frac{\partial p_{G}\left(\pi_{G g}^{2 t+1}(\mu), 1\right)}{\partial \mu} \frac{d \pi_{G g}\left(\pi_{G g}^{2 t}(\mu)\right)}{d \mu} \frac{d \pi_{G g}^{2 t}(\mu)}{d \mu}\right]
\end{gathered}
$$

for $\mu \geq \bar{\mu}$ because $y^{*}(\mu)=1$ for $\mu \geq \bar{\mu}$. By routine calculation, we get

$$
\begin{gathered}
(h-1) \frac{\partial p_{G}(\mu, 1)}{\partial \mu}+\left(h^{2}-\ell\right) \frac{\partial p_{G}\left(\pi_{G g}(\mu), 1\right)}{\partial \mu} \frac{d \pi_{G g}(\mu)}{d \mu} \\
=-\frac{h(1-h)(1-\ell)}{(1-(1-h) \mu)^{2}}+\frac{h^{2}\left(h^{2}-\ell\right)(1-\ell) \ell}{\left(\ell(1-\mu)+h^{2} \mu\right)^{2}},
\end{gathered}
$$

the derivative of which is

$$
-2(1-\ell) \frac{h(1-h)^{2}}{(1-(1-h) \mu)^{3}}-\frac{\ell\left(h^{3}-h \ell\right)^{2}}{\left(\ell(1-\mu)+h^{2} \mu\right)^{3}}<0 .
$$


If $h>\frac{1+\sqrt{1+4 \ell^{2}+4 \ell^{3}}}{2+2 \ell}$, it is routinely verified that (51) evaluated at $\mu=1$ is positive and thus, (51) is positive for all $\mu$ due to (52). This further implies that (50) is positive for all $\mu \geq \bar{\mu}$ and consequently, from (48),

$$
\frac{d V_{h}^{*}(\mu)}{d \mu} \geq \frac{d V_{\ell}^{*}(\mu)}{d \mu} \quad \forall \mu \geq \bar{\mu}
$$

when $\delta<1$ is sufficiently close to 1 , provided $h>\frac{1+\sqrt{1+4 \ell^{2}+4 \ell^{3}}}{2+2 \ell}$.

Next, let $\mu_{1}=\min \left\{\mu \mid \pi_{G g}(\mu) \geq \bar{\mu}\right.$ and $\left.\pi_{B b}\left(\mu, y^{*}(\mu)\right) \geq \bar{\mu}\right\}$ and consider $\mu \in\left[\mu_{1}, \bar{\mu}\right]$. Note that $\mu_{1}<\bar{\mu}$ due to Lemma 7 . Since

$$
\begin{aligned}
V_{h}^{*}(\mu) & =h p_{G}\left(\mu, y^{*}(\mu)\right)+\delta\left(h V_{h}^{*}\left(\pi_{G g}(\mu)\right)+(1-h) V_{h}^{*}\left(\pi_{B b}\left(\mu, y^{*}(\mu)\right)\right)\right) \text { and } \\
V_{\ell}^{*}(\mu) & =p_{G}\left(\mu, y^{*}(\mu)\right)+\delta\left(\ell V_{\ell}^{*}\left(\pi_{G g}(\mu)\right)+(1-\ell) V_{\ell}^{*}(0)\right)
\end{aligned}
$$

we deduce that $\frac{d V_{h}^{*}(\mu)}{d \mu}-\frac{d V_{\ell}^{*}(\mu)}{d \mu}$, which exists almost everywhere because both $V_{h}^{*}(\mu)$ and $V_{\ell}^{*}(\mu)$ are continuous and increasing, is equal to the derivative of

$$
(1-h)\left(\delta V_{h}^{*}\left(\pi_{B b}\left(\mu, y^{*}(\mu)\right)\right)-p_{G}\left(\mu, y^{*}(\mu)\right)\right)+\delta\left(h V_{h}^{*}\left(\pi_{G g}(\mu)\right)-\ell V_{\ell}^{*}\left(\pi_{G g}(\mu)\right)\right),
$$

which is positive due to (53) because $p_{G}\left(\mu, y^{*}(\mu)\right)=\delta\left(V_{\ell}^{*}\left(\pi_{B b}\left(\mu, y^{*}(\mu)\right)\right)-V_{\ell}^{*}(0)\right)$ for $\mu \leq \bar{\mu}$. Repeated application of analogous argument establishes that $\frac{d V_{h}^{*}(\mu)}{d \mu}>\frac{d V_{\ell}^{*}(\mu)}{d \mu}$ for all $\mu>0$ when $\delta<1$ is sufficiently close to 1 if $h>\frac{1+\sqrt{1+4 \ell^{2}+4 \ell^{3}}}{2+2 \ell}$.

Setting $\pi_{B b}(0,1)=\lim _{\mu \rightarrow 0} \pi_{B b}\left(\mu, y^{*}(\mu)\right)$ and $V_{h}^{*}(0)=\lim _{\mu \rightarrow 0} V_{h}^{*}(\mu)$, this implies that $h$-seller prefers to tell the truth upon drawing $q=b$ whenever $\ell$-seller is indifferent, i.e., when $\mu \in(0, \bar{\mu}]$. Then, $x^{*}(0, b)=0$ is optimal by continuity of $V_{h}^{*}, p_{G}\left(\mu, y^{*}(\mu)\right)$, $\pi_{G g}(\mu)$, and $\pi_{B b}\left(\mu, y^{*}(\mu)\right)$. Finally, optimality of $x^{*}(\mu, g)=0$ follows immediately from (13) as before.

Proof of Proposition 2. Since $\delta \Delta_{v_{o}}<\delta \Delta<1$, as before there is $\bar{\mu}^{\dagger} \in(0, \bar{\mu})$ such that $p_{G}\left(\bar{\mu}^{\dagger}, 1\right)=\delta\left(V_{v_{o}}^{\dagger}\left(\pi_{B b}\left(\bar{\mu}^{\dagger}, 1\right)\right)-v_{o}\right)$ so that $p_{G}(\mu, y)>\delta\left(V_{v_{o}}^{\dagger}\left(\pi_{B b}(\mu, y)\right)-v_{o}\right)$ for all $y \in[0,1]$ and, therefore, $y_{v_{o}}^{\dagger}(\mu)=1$ for all $\mu \geq \bar{\mu}^{\dagger}$.

Note that $y_{v_{o}}^{\dagger}$ is continuous by construction (which is analogous to (11)) and $y_{v_{o}}^{\dagger}(\mu) \in(0,1)$ for $\mu<\bar{\mu}^{\dagger}$. To reach a contradiction, suppose $y_{v_{o}}^{\dagger}\left(\mu^{\prime}\right)=y_{V_{\ell}^{*}}^{*}\left(\mu^{\prime}\right)$ for some $\mu^{\prime}<\bar{\mu}^{\dagger}$ and $y_{v_{o}}^{\dagger}(\mu)>y_{V_{\ell}^{*}}^{*}(\mu)$ for all $\mu \in\left(\mu^{\prime}, \bar{\mu}\right)$. Then,

$$
\begin{aligned}
\delta\left(V_{\ell}^{*}\left(\pi_{B b}\left(\mu^{\prime}, y_{V_{\ell}^{*}}^{*}\left(\mu^{\prime}\right)\right)\right)-V_{\ell}^{*}(0)\right) & =p_{G}\left(\mu^{\prime}, y_{V_{\ell}^{*}}^{*}\left(\mu^{\prime}\right)\right) \\
& =p_{G}\left(\mu^{\prime}, y_{v_{o}}^{\dagger}\left(\mu^{\prime}\right)\right)=\delta\left(V_{v_{o}}^{\dagger}\left(\pi_{B b}\left(\mu^{\prime}, y_{v_{o}}^{\dagger}\left(\mu^{\prime}\right)\right)\right)-v_{o}\right)
\end{aligned}
$$

and thus,

$$
V_{\ell}^{*}(\tilde{\mu})-V_{\ell}^{*}(0)=V_{v_{o}}^{\dagger}(\tilde{\mu})-v_{o} \quad \text { where } \quad \tilde{\mu}:=\pi_{B b}\left(\mu^{\prime}, y_{V_{\ell}^{*}}^{*}\left(\mu^{\prime}\right)\right)>\mu^{\prime}
$$

and the inequality is from Lemma 7. Furthermore, since

$$
\begin{aligned}
V_{\ell}^{*}(\tilde{\mu}) & =p_{G}\left(\tilde{\mu}, y_{V_{\ell}^{*}}^{*}(\tilde{\mu})\right)+\delta\left(\ell V_{\ell}^{*}\left(\pi_{G g}(\tilde{\mu})\right)+(1-\ell) V_{\ell}^{*}(0)\right) \text { and } \\
V_{v_{o}}^{\dagger}(\tilde{\mu}) & =p_{G}\left(\tilde{\mu}, y_{v_{o}}^{\dagger}(\tilde{\mu})\right)+\delta\left(\ell V_{v_{o}}^{\dagger}\left(\pi_{G g}(\tilde{\mu})\right)+(1-\ell) v_{o}\right)
\end{aligned}
$$


while $p_{G}\left(\tilde{\mu}, y_{V_{\ell}^{*}}^{*}(\tilde{\mu})\right) \geq p_{G}\left(\tilde{\mu}, y_{v_{o}}^{\dagger}(\tilde{\mu})\right),(54)-(56)$ would imply

$$
\delta \ell\left[\left(V_{\ell}^{*}\left(\pi_{G g}(\tilde{\mu})\right)-V_{\ell}^{*}(0)\right)-\left(V_{v_{o}}^{\dagger}\left(\pi_{G g}(\tilde{\mu})\right)-v_{o}\right)\right] \leq(\delta-1)\left(v_{o}-V_{\ell}^{*}(0)\right)<0 .
$$

Since $V_{\ell}^{*}(1)-V_{\ell}^{*}(0)=\Delta>\Delta_{v_{o}}=V_{v_{o}}^{\dagger}(1)-v_{o}$, there must exist $\mu^{\prime \prime} \in(\tilde{\mu}, 1)$ such that $V_{\ell}^{*}\left(\mu^{\prime \prime}\right)-V_{\ell}^{*}(0) \leq V_{v_{o}}^{\dagger}\left(\mu^{\prime \prime}\right)-v_{o}$ and $V_{\ell}^{*}(\mu)-V_{\ell}^{*}(0)>V_{v_{o}}^{\dagger}\left(\mu^{\prime}\right)-v_{o}$ for all $\mu>\mu^{\prime \prime}$. However, since $p_{G}\left(\mu^{\prime}, y_{V_{\ell}^{*}}^{*}\left(\mu^{\prime \prime}\right)\right) \geq p_{G}\left(\mu^{\prime}, y_{v_{o}}^{\dagger}\left(\mu^{\prime \prime}\right)\right)$ and $\pi_{G g}\left(\mu^{\prime \prime}\right)>\mu^{\prime}$, (55) and (56) evaluated at $\mu=\mu^{\prime \prime}$ imply that $V_{\ell}^{*}\left(\mu^{\prime \prime}\right)-\delta V_{\ell}^{*}(0)>V_{v_{o}}^{\dagger}\left(\mu^{\prime \prime}\right)-\delta v_{o}$ and consequently, $V_{\ell}^{*}\left(\mu^{\prime \prime}\right)-V_{\ell}^{*}(0)>V_{v_{o}}^{\dagger}\left(\mu^{\prime \prime}\right)-v_{o}$, contradicting the definition of $\mu^{\prime \prime}$.

Proof of Lemma 9. Since continuity under the sup norm requires uniform convergence, the possibility of a fixed point having unbounded derivative poses a potential problem. The bulk of the proof evolves around how to circumvent this problem. We start with two preliminary lemmas asserting that $p_{G}^{*}(\mu)$ is of bounded variation on $[\varepsilon, 1]$ for any $\varepsilon>0$ (Lemma A1) and consequently, so is the fixed point $V_{v_{o}}^{\dagger}$ (Lemma A2).

Lemma A1 For any $\varepsilon>0$ there exists $M_{\varepsilon}>0$ such that $\forall v_{o} \in\left[\frac{\ell}{1-\delta}, \frac{1}{1-\delta}\right), \forall V \in$ $\mathcal{F}_{v_{o}} \cap \mathcal{C}_{[0,1]}, \forall \mu$ and $\mu^{\prime} \in\left(\varepsilon, \bar{\mu}^{\dagger}\right)$,

$$
\frac{p_{G}\left(\mu^{\prime}, y_{V}^{\dagger}\left(\mu^{\prime}\right)\right)-p_{G}\left(\mu, y_{V}^{\dagger}(\mu)\right)}{\mu^{\prime}-\mu} \leq M_{\varepsilon}
$$

Proof. Note from (3) that we can find $k>0$ such that $\frac{\partial p_{G}}{\partial \mu}>0$ is bounded above uniformly by $k$, and $\frac{\partial p_{G}}{\partial y}<0$ is bounded below uniformly by $-k$. Suppose $\mu<\mu^{\prime}$ without loss of generality.

If $y_{V}^{\dagger}\left(\mu^{\prime}\right) \geq y_{V}^{\dagger}(\mu)$, then $\frac{p_{G}\left(\mu^{\prime}, y_{V}^{\dagger}\left(\mu^{\prime}\right)\right)-p_{G}\left(\mu, y_{V}^{\dagger}(\mu)\right)}{\mu^{\prime}-\mu}<k$ because $p_{G}$ decreases in $y$, proving (58).

Now suppose that $y_{V}^{\dagger}\left(\mu^{\prime}\right)<y_{V}^{\dagger}(\mu)$. Note that one can find $k_{\varepsilon}, \tilde{k}_{\varepsilon}>0$ such that

$$
\begin{aligned}
& \frac{\partial \pi_{B b}(\mu, y)}{\partial \mu}=\frac{(1-h)(1-\ell)(1-y)}{[\mu(1-h)+(1-\mu)(1-\ell)(1-y)]^{2}}<k_{\varepsilon} \\
& \frac{\partial \pi_{B b}(\mu, y)}{\partial y}=\frac{(1-h)(1-\ell)(1-\mu) \mu}{[\mu(1-h)+(1-\mu)(1-\ell)(1-y)]^{2}}>\tilde{k}_{\varepsilon}
\end{aligned}
$$

for all $\mu>\varepsilon$ and $y \in[0,1]$. Thus, recalling that $\pi_{B b}\left(\mu, y_{V}^{\dagger}(\mu)\right)$ is nondecreasing, we deduce that

$$
0 \leq \pi_{B b}\left(\mu^{\prime}, y_{V}^{\dagger}\left(\mu^{\prime}\right)\right)-\pi_{B b}\left(\mu, y_{V}^{\dagger}(\mu)\right)<k_{\varepsilon}\left(\mu^{\prime}-\mu\right)+\tilde{k}_{\varepsilon}\left(y_{V}^{\dagger}\left(\mu^{\prime}\right)-y_{V}^{\dagger}(\mu)\right),
$$

using the facts that $y_{V}^{\dagger}\left(\mu^{\prime}\right)<y_{V}^{\dagger}(\mu)$ and $\mu<\mu^{\prime}$, and consequently,

$$
y_{V}^{\dagger}\left(\mu^{\prime}\right)-y_{V}^{\dagger}(\mu)>-\frac{k_{\varepsilon}}{\tilde{k}_{\varepsilon}}\left(\mu^{\prime}-\mu\right) \text {. }
$$


Therefore, we have

$$
\begin{aligned}
p_{G}\left(\mu^{\prime}, y_{V}^{\dagger}\left(\mu^{\prime}\right)\right)-p_{G}\left(\mu, y_{V}^{\dagger}(\mu)\right) & <k\left(\mu^{\prime}-\mu\right)-k\left(y_{V}^{\dagger}\left(\mu^{\prime}\right)-y_{V}^{\dagger}(\mu)\right) \\
& <k\left(1+\frac{k_{\varepsilon}}{\tilde{k}_{\varepsilon}}\right)\left(\mu^{\prime}-\mu\right) .
\end{aligned}
$$

We complete the proof by setting $M_{\varepsilon}=k\left(1+\frac{k_{\varepsilon}}{\tilde{k}_{\varepsilon}}\right)$.

Lemma A2 For any $\varepsilon>0$ and $v_{o} \in\left(\frac{\ell}{1-\delta}, \frac{1}{1-\delta}\right)$,

$$
D^{+} V_{v_{o}}^{\dagger}(\mu):=\lim _{\mu^{\prime} \downarrow \mu} \sup \frac{V_{v_{o}}^{\dagger}\left(\mu^{\prime}\right)-V_{v_{o}}^{\dagger}(\mu)}{\mu^{\prime}-\mu} \leq \frac{M_{\varepsilon}}{1-\delta h} \quad \text { if } \mu>\varepsilon,
$$

where $V_{v_{o}}^{\dagger}$ is the fixed point of $T_{v_{o}}$.

Proof. For given $v_{o}$ there exists $\underline{\mu}>0$ defined by $V_{v_{o}}^{\dagger}\left(\pi_{G g}(\underline{\mu})\right)=v_{o}$, so that

$$
V_{v_{o}}^{\dagger}(\mu)=\left\{\begin{array}{l}
p_{G}\left(\mu, y_{v_{o}}^{\dagger}(\mu)\right)+\delta v_{o} \quad \text { if } \mu \leq \underline{\mu} \\
p_{G}\left(\mu, y_{v_{o}}^{\dagger}(\mu)\right)+\delta\left(\ell V_{v_{o}}^{\dagger}\left(\pi_{G g}(\mu)\right)+(1-\ell) v_{o}\right) \quad \text { if } \mu \geq \underline{\mu} .
\end{array}\right.
$$

To reach a contradiction, suppose that for any $K>0$ one can find $\mu_{1}>\varepsilon$ such that $D^{+} V_{v_{o}}^{\dagger}\left(\mu_{1}\right)>K$. Then, since $\pi_{G g}(\mu)$ is differentiable and $\frac{\ell}{h} \leq \frac{\partial \pi_{G g}(\mu)}{\partial \mu} \leq \frac{h}{\ell}$, (58) and (60) would imply that $\mu_{1}>\mu$ when $K$ is sufficiently large and that one can construct a sequence $\mu_{n} \rightarrow 1$ where $\mu_{n}=\pi_{G g}\left(\mu_{n-1}\right)$. Since there is $\tau<\infty$ such that $\pi_{G g}^{\tau}(\mu)>\bar{\mu}^{\dagger}$ for any $\mu>\varepsilon$, by choosing $K$ arbitrarily large, one can ensure that $D^{+} V_{v_{o}}^{\dagger}\left(\pi_{G g}^{\tau}(\mu)\right)$ is arbitrarily large. But, this is impossible because $D^{+} V_{v_{o}}^{\dagger}(\mu)$ is bounded for $\mu>\bar{\mu}^{\dagger}$ as can be verified from

$$
V_{v_{o}}^{\dagger}(\mu)=\left[\sum_{t=0}^{\infty} \ell^{t} \delta^{t} p_{G}\left(\pi_{G g}^{t}(\mu), 1\right)\right]+\delta v_{o}(1-\ell) \sum_{t=0}^{\infty} \ell^{t} \delta^{t}
$$

a formula adapted from (44) for $V_{v_{o}}^{\dagger}(\mu)$ for $\mu>\bar{\mu}^{\dagger}$. Hence, we conclude that $D^{+} V_{v_{o}}^{\dagger}(\mu)$ is uniformly bounded for $\mu>\varepsilon$ and thus, (58) and (60) imply

$$
\begin{aligned}
D^{+} V_{v_{o}}^{\dagger}(\mu) & \leq M_{\varepsilon}+\ell \delta\left(\sup _{\mu>\varepsilon} D^{+} V_{v_{o}}^{\dagger}(\mu)\right)\left(\max _{\mu} \frac{\partial \pi_{G g}(\mu)}{\partial \mu}\right) \\
& \leq M_{\varepsilon}+h \delta\left(\sup _{\mu>\varepsilon} D^{+} V_{v_{o}}^{\dagger}(\mu)\right)
\end{aligned}
$$

for $\mu>\varepsilon$. Thus, $D^{+} V_{v_{o}}^{\dagger}(\mu) \leq \frac{M_{\varepsilon}}{1-\delta h}$ if $\mu>\varepsilon$.

Next, choose $v_{o} \in\left(\frac{\ell}{1-\delta}, \frac{1}{1-\delta}\right)$. Notice that for a sufficiently small $\eta>0$, in particular smaller than $v_{o}-\frac{\ell}{1-\delta}$, the operator $T_{v_{o}}$ can be extended to $\mathcal{F}_{v_{o}}^{\eta} \cap \mathcal{C}_{[0,1]}$ where $\mathcal{F}_{v_{o}}^{\eta}:=\cup_{v_{o}-\eta \leq v \leq v_{o}+\eta} \mathcal{F}_{v}$. As an intermediate step, we need 
Lemma A3 For $v_{o} \in\left(\frac{\ell}{1-\delta}, \frac{1}{1-\delta}\right)$, the operator

$$
T_{v_{o}}: \mathcal{F}_{v_{o}}^{\eta} \cap \mathcal{C}_{[0,1]} \rightarrow \mathcal{C}_{[0,1]} \text { is continuous in sup norm }
$$

Proof. Consider $V, V^{\prime} \in \mathcal{F}_{v_{o}}^{\eta} \cap \mathcal{C}_{[0,1]}$ such that $\max _{\mu \in[0,1]}\left|V^{\prime}(\mu)-V(\mu)\right|<\epsilon$. Since $y_{V}^{\dagger}(\mu)$ and $y_{V^{\prime}}^{\dagger}(\mu)$ are, by construction, the solutions to

$$
p_{G}(\mu, y)=\delta\left(\max \left\{v_{o}, V\left(\pi_{B b}(\mu, y)\right)\right\}-v_{o}\right)
$$

and the same equation with $V^{\prime}$ instead of $V$, respectively, $\left|p_{G}\left(\mu, y_{V^{\prime}}^{\dagger}(\mu)\right)-p_{G}\left(\mu, y_{V}^{\dagger}(\mu)\right)\right|<$ $\epsilon$. From (21), therefore, we deduce that

$$
\max _{\mu \in[0,1]}\left|T_{v_{o}}\left(V^{\prime}\right)(\mu)-T_{v_{o}}(V)(\mu)\right|<\epsilon+\delta \epsilon,
$$

which establishes (62).

Given $v_{o} \in\left(\frac{\ell}{1-\delta}, \frac{1}{1-\delta}\right)$ and $\eta$ small as specified above, consider small $|\kappa|<\eta / 2$ and any $V \in \mathcal{F}_{v_{o}}^{\eta} \cap \mathcal{F}_{v_{o}+\kappa}^{\eta} \cap \mathcal{C}_{[0,1]}$. By (63), the value of $p_{G}\left(\mu, y_{V}^{\dagger}(\mu)\right)$ differs when calculated for $T_{v_{o}}$ and when calculated for $T_{v_{o}+\kappa}$, and the difference is at most $\delta \kappa$. Thus, from $(21)$

$$
T_{v_{o}}(V)(\mu)-2|\delta \kappa| \leq T_{v_{o}+\kappa}(V)(\mu) \leq T_{v_{o}}(V)(\mu)+2|\delta \kappa| \quad \forall \mu \in[0,1] .
$$

In particular, observe that

$$
T_{v_{o}}\left(V_{v_{o}+\kappa}^{\dagger}\right)(\mu)-2|\delta \kappa| \leq T_{v_{o}+\kappa}\left(V_{v_{o}+\kappa}^{\dagger}\right)(\mu)=V_{v_{o}+\kappa}^{\dagger}(\mu) \leq T_{v_{o}}\left(V_{v_{o}+\kappa}^{\dagger}\right)(\mu)+2|\delta \kappa| .
$$

Finally, to prove continuity of $\psi$ at $v_{o}$, we decompose the argument into two parts: First, we prove uniform convergence of functions $\psi\left(v_{o}+\kappa\right)=V_{v_{o}+\kappa}^{\dagger}$ to $\psi\left(v_{o}\right)=V_{v_{o}}^{\dagger}$ as $\kappa \rightarrow 0$ on intervals $[\varepsilon, 1]$, then do the same separately on $[0,2 \varepsilon]$. The continuity will be established by combining the two parts.

We know from Lemma A2 that on the interval $[\varepsilon, 1]$, the function $V_{v_{o}+\kappa}^{\dagger}$ is $K_{\varepsilon^{-}}$ Liptchitz where $K_{\varepsilon}=\frac{M_{\varepsilon}}{1-\delta h}$. Then from Ascoli-Arzelà Theorem (see Royden (1988)), the subset consisting of all $K_{\varepsilon}$-Lipschitz function of $\mathcal{F}_{v_{o}}^{\eta}$ is compact under the sup norm. Hence, there exists a sequence of fixed points $V_{v_{o}+\kappa}^{\dagger}$ such that, when restricted to the domain $[\varepsilon, 1]$, it converges as $\kappa \rightarrow 0$ to a limit, denoted by $W_{v_{o}}^{[\varepsilon, 1]}$, where $W_{v_{o}}^{[\varepsilon, 1]}$ is continuous on $[\varepsilon, 1]$ and

(c0) $V_{v_{o}+\kappa}^{\dagger} \stackrel{\text { unif }}{\longrightarrow} W_{v_{o}}^{[\varepsilon, 1]}$ under the sup norm on $[\varepsilon, 1]$ for any $\varepsilon>0$.

Let $V_{v_{o}+\kappa}^{\dagger[\varepsilon, 1]}$ denote $V_{v_{o}+\kappa}^{\dagger}$ restricted on $[\varepsilon, 1]$ and let $\widetilde{V}_{v_{o}+\kappa}^{\dagger[\varepsilon, 1]}$ denote the continuous linear extension of $V_{v_{o}+\kappa}^{\dagger[\varepsilon, 1]}$ on $[0, \varepsilon]$. Then, by (62) and (64),

$$
T_{v_{o}}\left(\lim _{\kappa \rightarrow 0} \widetilde{V}_{v_{o}+\kappa}^{\dagger[\varepsilon, 1]}\right)(\mu) \leq \lim _{\kappa \rightarrow 0} T_{v_{o}+\kappa}\left(\widetilde{V}_{v_{o}+\kappa}^{\dagger[\varepsilon, 1]}\right)(\mu) \leq T_{v_{o}}\left(\lim _{\kappa \rightarrow 0} \widetilde{V}_{v_{o}+\kappa}^{\dagger[\varepsilon, 1]}\right)(\mu) .
$$


Note that $T_{v_{o}}\left(\lim _{\kappa \rightarrow 0} \widetilde{V}_{v_{o}+\kappa}^{\dagger[\varepsilon, 1]}\right)(\mu)$ for each $\mu$ is fully determined by $\lim _{\kappa \rightarrow 0} \widetilde{V}_{v_{o}+\kappa}^{\dagger[\varepsilon, 1]}$ restricted on $[\mu, 1]$ according to (21), and the same is true for $T_{v_{o}+\kappa}\left(\widetilde{V}_{v_{o}+\kappa}^{\dagger[\varepsilon, 1]}\right)$. Since $\widetilde{V}_{v_{o}+\kappa}^{\dagger[\varepsilon, 1]}=V_{v_{o}+\kappa}^{\dagger}$ on $[\varepsilon, 1]$ by definition, therefore, $(\mathrm{c} 0)$ and (65) imply that

$$
T_{v_{o}}\left(\widetilde{W}_{v_{o}}^{[\varepsilon, 1]}\right)(\mu) \leq \widetilde{W}_{v_{o}}^{[\varepsilon, 1]}(\mu) \leq T_{v_{o}}\left(\widetilde{W}_{v_{o}}^{[\varepsilon, 1]}\right)(\mu) \text { for all } \mu \in[\varepsilon, 1]
$$

where $\widetilde{W}_{v_{o}}^{[\varepsilon, 1]}$ is the continuous linear extension of $W_{v_{o}}^{[\varepsilon, 1]}$ on $[0, \varepsilon]$. Since $\varepsilon>0$ is arbitrary and $V_{v_{o}}^{\dagger}$ is the only function $V$ that satisfies $T_{v_{o}}(V)(\mu)=V(\mu)$ on $[\varepsilon, 1]$ for all $\varepsilon \in(0,1)$ by uniqueness of the fixed point of $T_{v_{o}}$, it further follows that $\widetilde{W}_{v_{o}}^{[\varepsilon, 1]}=V_{v_{o}}^{\dagger}$ on $[\varepsilon, 1]$, i.e., $W_{v_{o}}^{[\varepsilon, 1]}$ coincides with $V_{v_{o}}^{\dagger}$ on $[\varepsilon, 1]$. From $(\mathrm{c} 0)$, therefore,

(c1) $V_{v_{o}+\kappa}^{\dagger} \stackrel{\text { unif }}{\longrightarrow} V_{v_{o}}^{\dagger}$ under the sup norm on $[\varepsilon, 1]$ for any $\varepsilon>0$.

Note, however, that this is not sufficient for uniform convergence on $[0,1]$. Hence, choose $\breve{\mu}>0$ such that $V_{v_{o}}^{\dagger}\left(\pi_{G g}(\breve{\mu})\right)<v_{o}$. Then, because $V_{v_{o}+\kappa}^{\dagger}$ converges to $V_{v_{o}}^{\dagger}$ under the sup norm on $\left[\frac{\breve{\mu}}{2}, 1\right]$ by $(\mathrm{c} 1)$, we have $V_{v_{o}+\kappa}^{\dagger}\left(\pi_{G g}(\breve{\mu})\right)<v_{o}+\kappa$ for sufficiently small $\kappa$. But this implies that $V_{v_{o}+\kappa}^{\dagger}\left(\pi_{G g}(\mu)\right)<v_{o}+\kappa$ for all $\mu \leq \breve{\mu}$ for sufficiently small $\kappa$, and consequently, $V_{v_{o}+\kappa}^{\dagger}(\mu)=p_{G}(\mu, 1)+\delta\left(v_{o}+\kappa\right)$ on $[0, \breve{\mu}]$, which converges uniformly to $V_{v_{o}}^{\dagger}(\mu)=p_{G}(\mu, 1)+\delta v_{o}$. Thus,

(c2) $V_{v_{o}+\kappa}^{\dagger} \stackrel{\text { unif }}{\longrightarrow} V_{v_{o}}^{\dagger}$ under the sup norm on $[0, \breve{\mu}]$.

Combining (c1) and (c2), we obtain uniform convergence under the sup norm on the entire domain $[0,1]$, which proves continuity of $\psi$ at $v_{o}$.

\section{References}

Bajari, P., and A. Hortacsu, (2004), "Economic Insights from Internet Auctions," Journal of Economic Literature, 42, 457-486.

Bar-Isaac, H. (2003), "Reputation and Survival: learning in a dynamic signalling model", Review of Economic Studies, 70, 231-251.

Bar-Isaac H. and S. Tadelis, (2008), "Seller Reputation", Foundations and Trends in Microeconomics, 4, 273-351

Benabou, R. and G. Laroque (1992), "Using Privileged Information to Manipulate Markets: Insiders, Gurus and Credibility," Quarterly Journal of Economics, 107, 921-958.

Billingsley, P. (1999), Convergence of Probability Measures, 2nd ed., John Wiley \& Sons, Inc.

Cabral, L., and A. Hortacsu, (2008), "The Dynamics of Seller Reputation: Theory and Evidence from eBay," mimeo, New York University. 
Canals-Cerda, J. (2008), "The Value of a Good Reputation Online: An Application to Art Auctions," mimeo, Federal Reserve Bank of Philadelphia

Cripps, M., Mailath, G., and L. Samuelson (2004), "Imperfect Monitoring and Impermanent Reputations," Econometrica, 72, 407-432.

Dellarocas, C. (2003). "The Digitization of Word of Mouth: Promise and Challenges of Online Feedback Mechanisms," Management Science, 49, 1407-1424.

Dellarocas, C. (2006), "Reputation Mechanisms", Handbook on Economics and Information Systems (T. Hendershott, ed.), Elsevier Publishing.

Diamond, D. (1989), "Reputation Acquisition in Debt Market", Journal of Political Economy, 97, 828-862.

Ely, J., and J. Valimaki (2003), "Bad Reputation," Quarterly Journal of Economics, 118, 785-814.

Fan, K. (1952), "Fixed point and minimax theorems in locally convex topological linear spaces," Proc. Nat. Acad. Sci. U.S.A., 38, 121-126.

Fudenberg, D., and D. Levine (1989), "Reputation and equilibrium selection in games with a patient player," Econometrica, 57, 759-778.

Glicksberg, I. L. (1952), "A further generalization of the Kakutani fixed point theorem, with application to Nash equilibrium points," Proc. Amer. Math. Soc., 3, 170-174.

Jin, G. and A. Kato (2006), "Price, Quality and Reputation: Evidence from an Online Field Experiment," RAND Journal of Economics, 37, 983-1005.

Kreps, D. and R. Wilson (1982), "Reputation and Imperfect Information," Journal of Economic Theory, 27, 245-252.

Mailath, G. and L. Samuelson (2001), "Who Wants a Good Reputation," Review of Economic Studies, 68, 415-441.

Mailath, G. and L. Samuelson (2006), Repeated Games and Reputations: Long-Run Relationships, Oxford University Press.

Mathis,J., McAndrews, J. and J.C. Rochet (2009), "Rating the raters: are reputation concerns powerful enough to discipline rating agencies?", mimeo, Toulouse School of Economics.

McLennan, A. and I.-U. Park (2007), "The Market for Liars: Reputation and Auditor Honesty," mimeo, University of Bristol.

Milgrom, P. and D.J. Roberts (1982), "Predation, Reputation and Entry Deterrence," Journal of Economic Theory, 27, 280-312.

Morris, S. (2001), "Political Correctness," Journal of Political Economy, 109, 231-265.

Ottaviani, M. and P. Sorensen (2001) "Information aggregation in debate: who should speak first", Journal of Public Economics, 81, 393-42.

Ottaviani, M. and P. Sorensen (2006), "Reputational cheap talk," RAND Journal of Economics, 37, 155-175.

Park, I.-U. (2005), "Cheap-talk referrals of differentiated experts in repeated relationships," RAND Journal of Economics, 36, 391-441.

Sobel, J. (1985), "A Theory of Credibility," Review of Economic Studies, 52, 557-573.

Royden H.L. (1988), Real Analysis, 3rd ed., Macmillan Publishing Company, New York. 